\title{
Computer simulation of coherent Doppler lidar measurement of wind velocity and retrieval of turbulent wind statistics
}

\author{
V. A. Banakh \\ Siberian Branch-Russian Academy of Science \\ Institute of Atmospheric Optics \\ Akademicheskii prospekt, 1 \\ Tomsk 634055, Russia \\ E-mail: banakh@iao.ru
}

\author{
C. Werner \\ DLR Institute of Atmospheric Physics \\ D-82234 Wessling, Germany \\ E-mail: christian.werner@DLR.de
}

\begin{abstract}
We describe the algorithms for the simulation of $\mathrm{cw}$ and pulsed coherent Doppler wind lidar operation in a turbulent atmosphere and the methods to estimate the mean wind and the parameters of small-scale wind turbulence from lidar data. The algorithms are realized as LabView computer codes, which include parts simulating the atmospheric turbulent wind fields based on the Karman model, lidar signal, and data processing. The errors for lidar measurement of turbulent parameters and the retrieval of the wind vertical profiles are estimated based on a computer simulation of the coherent Doppler lidar system operation. (c) 2005 Society of Photo-Optical Instrumentation Engineers. [DOI: 10.1117/1.1955167]
\end{abstract}

Subject terms: wind coherent Doppler lidar; turbulence; statistics; simulation.

Paper SS040434R received Jul. 5, 2004; revised manuscript received Nov. 26, 2004; accepted for publication Nov. 29, 2004; published online Jul. 15, 2005.

\section{Introduction}

Coherent Doppler lidars (CDLs) are finding expanding applications in study of wind fields in the atmosphere. The existing CDL systems ${ }^{1-9}$ enable measurements of the wind speed and direction with high spatial resolution from both the ground and the air. Some National Aeronautics and Space Administration (NASA) and European Space Agency (ESA) projects ${ }^{10,11}$ have considered the feasibility of using CDLs to measure the wind speed and direction from space.

The data of lidar wind measurements can be used to retrieve wind turbulence parameters, such as, for example, the dissipation rate of the turbulent energy, the variance of the wind velocity, the outer scale of turbulence, and the vertical turbulent momentum flux. The first results of CDL measurements of the wind turbulence parameters are presented in Refs. 12-14, and the results of the following experiments on the investigation of turbulence and atmospheric air flows with the use of CDLs can be found, in particular, in Refs. 15-26.

At the same time, the accuracy of Doppler lidar measurements of the wind and the wind turbulence parameters, as well as selection of the optimal geometry of sensing and the measurement time when planning lidar experiments have not yet been thoroughly studied. Computer simulation of the Doppler lidar operation in a turbulent atmosphere opens wide possibilities for solution of these problems, since it enables simulation of field experiments for given atmospheric conditions. Actually, if we simulate the turbulent field of the wind and the lidar return signal for certain vertical profiles of mean wind and parameters of atmospheric turbulence and properly process the model lidar data, then we can always compare the obtained lidar estimates of the wind speed and direction and the parameters

0091-3286/2005/\$22.00 @ 2005 SPIE of wind turbulence with those preset when simulating the wind field and thus determine the accuracy of lidar measurements.

The fundamental contribution to the methods of simulation of a CDL signal in a turbulent atmosphere and understanding the effect of wind turbulence on the CDL performance is found in Refs. 27-38. The accuracy of CDL radial velocity estimates, based on simulation, is discussed in Refs. 29, 31, 32, 34, 35, 37, and 38. The feasibility and accuracy of retrieval of spatial velocity statistics from CDL measurement data is considered both theoretically and experimentally in Refs. 17,33 , and 36 .

This paper reviews the methods for computer simulation of lidar systems developed at the Institute of Atmospheric Optics, Siberian Branch of the Russian Academy of Sciences (SB RAS) (Russia) in cooperation with the DLR Institute of Atmospheric Physics (Germany). These methods enable estimating the accuracy of lidar measurements of turbulent wind fields in the atmosphere based on the model lidar data. Academician V. E. Zuev, to whose memory this special issue of Optical Engineering is dedicated was initiator of that Russian-German cooperation and supported this study during many years.

Section 2 presents the algorithms for simulating a return signal of the scanning continuous-wave (cw) $\mathrm{CO}_{2} \mathrm{CDL}$ and estimating the mean wind vector and wind turbulence parameters from the model lidar data. The accuracy of retrieval of the turbulence parameters from the simulated $\mathrm{cw}$ $\mathrm{CO}_{2} \mathrm{CDL}$ velocity estimates is assessed for different numbers of scans.

Section 3 summarizes the results concerning the methods of retrieving vertical profiles of the mean wind velocity and direction based on the simulated data of a space-borne scanning pulsed CDL. The accuracy of wind profile retrieval is compared for two methods: (1) from an array of single estimates of the radial velocity and (2) from the accumulated Doppler spectra. The altitudes up to which the 
wind speed and direction can be retrieved with acceptable accuracy from measurements of a space-borne scanning pulsed 2- $\mu \mathrm{m}$ CDL are estimated.

In Sec. 4, based on the simulated pulsed CDL data, we discuss the methods and algorithms for estimation of the wind turbulence parameters from the spatial structure function of wind velocity for the cases of both short separations between measurement points $(2-\mu \mathrm{m} C D L)$ and long ones (10.6- $\mu \mathrm{m} \mathrm{CDL).} \mathrm{The} \mathrm{errors} \mathrm{in} \mathrm{estimating} \mathrm{the} \mathrm{turbulent} \mathrm{pa-}$ rameters from the lidar data are determined as functions of the signal storage time.

\section{Prediction of the Accuracy of cw CDL Measurement of Wind and Turbulence Parameters in the Atmospheric Boundary Layer}

Let the $\mathrm{cw} \mathrm{CO}_{2}$ Doppler lidar be placed in the plane $z=0$. A laser beam with the Gaussian amplitude profile and effective radius $a_{0}$ at the output from a transceiving telescope propagates along the $z$ axis of the Cartesian coordinates $\mathbf{r}$ $=\{z, x, y\}$. It is focused at a distance $R$ from the lidar. Radiation backscattered by aerosol particles is collected by the telescope and, together with a reference beam of the same wavelength $\lambda$ as that of the sounding beam, is incident on a photodetector. The valid component $j_{s}$ of the current in a photodetector circuit is described by the expression of the complex form ${ }^{39-41}$

$j_{s}(t)=B \sum_{l=1}^{n_{s}} a_{l} E^{2}\left[\mathbf{r}_{l}(t)\right] \exp \left[2 j k z_{l}(t)\right]$,

where $B=G$ e $\eta_{Q}(h \nu)^{-1} \lambda\left(P_{L} / P_{T}\right)^{1 / 2}, G$ is the gain, $e$ is the electronic charge, $\eta_{Q}$ is the detector quantum efficiency, $h \nu$ is the photon energy, $\lambda$ is the wavelength of the sensing beam, $P_{L}$ is the power of the reference beam, $P_{T}$ is the power of the sensing beam, $n_{s}$ is the number of aerosol particles contributing to $j_{s}(t), a_{l}$ is the backscattering amplitude of the $l$ ' th particle located at time $t$ at the point

$\mathbf{r}_{l}(t)=\mathbf{r}_{l_{0}}+\int_{0}^{t} \mathrm{~d} t^{\prime} \mathbf{U}^{L}\left(\mathbf{r}_{l_{0}}, t^{\prime}\right)$

where $\mathbf{r}_{l_{0}}$ is a point of the initial location of the $l$ ' th particle, and $\mathbf{U}^{L}=\left\{U_{z}^{L}, U_{x}^{L}, U_{y}^{L}\right\}$ is the vector of particle velocity (Lagrange wind velocity). The Lagrange velocity and the velocity $\mathbf{U}(\mathbf{r}, t)=\left\{U_{z}, U_{x}, U_{y}\right\}$ at a fixed point (Euler wind velocity) are related by the following equation:

$\mathbf{U}^{L}\left(\mathbf{r}_{l_{0}}, t\right)=\mathbf{U}\left[\mathbf{r}_{l}(t), t\right]$.

The function

$E(\mathbf{r})=\frac{\left(P_{T} / \pi\right)^{1 / 2}}{a_{0} g(z)} \exp \left[-\left(1+j \frac{k_{0} a_{0}^{2}}{R}\right) \frac{\rho^{2}}{2 a_{0}^{2} g(z)}\right]$,

is the complex field amplitude of the sounding beam, $g(z)$ $=(1-z / R)+j z /\left(k_{0} a_{0}^{2}\right), k_{0}=2 \pi / \lambda, \rho=\{x, y\}$ is a $2-\mathrm{D}$ vector perpendicular to $z$ axis, and $j$ is the imaginary unit.

The initial radius of sounding beam $a_{0}$ coincides with the effective radius of a Gaussian beam $a_{1}$ of complex am-

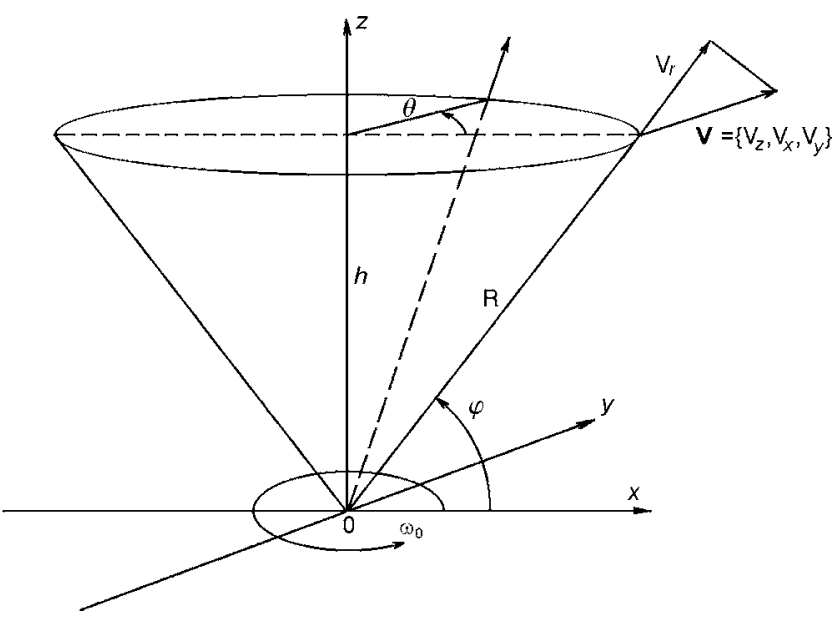

Fig. 1 Geometry of lidar with conical scanning.

plitude $E(0, \rho)=\exp \left[-\left(\rho^{2} / 2 a_{1}^{2}\right)-j k_{0}\left(\rho^{2} / R\right)\right] \quad\left(a_{0} \approx a_{1}\right)$, if the radius of the aperture of tranceiving telescope $a_{t}$ is much greater than $a_{1}$. If the condition $a_{t} \gg a_{1}$ is violated, then we should take into account influence of the finitness of the telescope aperture. This can be done easily for a Gaussian approximation of the telescope amplitude coefficient. In this case, we have $a_{0}=a_{1} /\left(1+a_{1}^{2} / 2 a_{t}^{2}\right)^{1 / 2}$.

The characteristic temporal scales of fluctuations of photocurrent $j_{s}(t)$ are less significant than the microscale of temporal variations of the Lagrange wind velocity. This enables us to use the approximation $\mathbf{r}_{l}(t)=\mathbf{r}_{l}+t U\left(\mathbf{r}_{l}\right)$, where $\mathbf{r}_{l} \equiv \mathbf{r}_{l_{0}}, \mathbf{r}_{l}=\left\{z_{l}, \rho_{l}\right\}$, in Eq. (1).

Assume that received signal $j_{s}(t)$ is fed to a spectrum analyzer where it passes through a linear bandpass filter with narrow bandwidth $\Delta f$. Then a squared modulus of the signal as it passes through such a filter $J_{s}(f, t)$ at its central frequency $f$ is averaged over integration period $t_{0}$ to obtain the estimates of spectral power density $\hat{W}(f, t)$. The expressions for $J_{s}(f, t)$ and $\hat{W}(f, t)$ are written as

$J_{s}(f, t)=\Delta f \int_{-1 / 2 \Delta f}^{1 / 2 \Delta f} \mathrm{~d} t^{\prime} j_{s}\left(t+t^{\prime}\right) \exp \left(-2 \pi j f t^{\prime}\right)$,

$\hat{W}(f, t)=\frac{1}{\Delta f} \frac{1}{t_{0}} \int_{t-t_{0}}^{t} \mathrm{~d} t^{\prime}\left|J_{s}\left(f, t^{\prime}\right)\right|^{2}$.

As an estimate of Doppler velocity the following formula can be used:

$\hat{V}_{D}(t)=\hat{S}^{-1}(t)(\lambda / 2) \int_{-\infty}^{\infty} \mathrm{d} f f \hat{W}(f, t)$,

where $S=\left\langle\left|j_{S}\right|^{2}\right\rangle=B^{2} \beta_{\pi} \int \mathrm{d}^{3} r|E(\mathbf{r})|^{4}$, the angle brackets indicate ensemble averaging, $\beta_{\pi}=\overline{\alpha_{l}^{2}} \rho_{c}$ is the backscattering coefficient, $\rho_{c}$ is the concentration of particles, and $\overline{\alpha_{l}^{2}}$ is a mean section of the particle backscattering.

Figure 1 shows the geometry of conical scanning by a laser beam in measurements with a cw Doppler lidar. The beam is inclined to the horizontal plane $\{x, y\}$ by the angle 
$\varphi$, and rotates around the vertical $z$ axis with the angular velocity $\omega_{0}$. The azimuthal scanning angle at the time $t$ is denoted by $\theta$ in the figure, that is, $\theta=\omega_{0} t$. The sensed volume centered at the height $h \approx R \sin \varphi$ is formed by focusing the beam at a distance $R$.

From a single measurement at the time moment $t$ $=\theta / \omega_{0}$ we can estimate only the radial component of the wind velocity vector $V_{r}\left(z^{\prime}, \theta\right)$, which is related to the wind components $\mathbf{V}(\mathbf{r}, t)=\left\{V_{z}, V_{x}, V_{y}\right\}$ at a fixed point $\mathbf{r}$ at the time $t$ as

$V_{r}\left(z^{\prime}, \theta\right)=\mathbf{s} \mathbf{V}\left(z^{\prime} \mathbf{s}, \theta / \omega_{0}\right)$,

where $z^{\prime}=|\mathbf{r}|$ is the distance from the lidar to the observation point $\mathbf{S}=\{\sin \varphi, \cos \varphi \cos \theta, \cos \varphi \sin \theta\}$.

During the scanning, the Doppler power spectra of the detected signal $W\left(f, m t_{0}\right)$, where $m=1,2,3, \ldots$ is the spectrum number, are recorded successively with the time interval $t_{0}$ [Eq. (4)]. One such spectrum is estimated through averaging of individual (unsmoothed) spectra recorded for the time $t_{s}=1 / \Delta f$, where $\Delta f$ is the frequency resolution. To decrease fluctuations of the estimated spectrum due to both random variations of the field of the scattered wave and the system noise, from hundred to thousand of such averagings are usually used. For example, for $\Delta f=20 \mathrm{kHz}$ and the number of averagings $N_{a}=1000$ the time for recording of a single spectrum is $t_{0}=N_{a} / \Delta f=50 \mathrm{~ms}$.

Taking into account the Doppler relation $\Delta f=(2 / \lambda) \Delta V$, where $\Delta V$ is the velocity resolution and $\lambda$ is the wavelength, the Doppler spectrum can be presented in the form

$W(k \Delta V, m \Delta \theta)=W_{s}(k \Delta V, m \Delta \theta)+W_{n}(k \Delta V, m \Delta \theta)$,

where $k=0,1,2, \ldots$ is the number of the spectral channel, and $\Delta \theta=\omega_{0} t_{0}$ is the resolution in the azimuthal angle. The first term in Eq. (7) is the power spectrum fully averaged over its fluctuations under the assumption that the distribution of the velocities of scattered particles in the sensed volume are unchanged (frozen turbulence). The second term represents fluctuations of the estimated spectrum determined by the SNR and the averaging time $t_{0}$.

When the number of efficiently scattering particles present in the sensed volume is rather great, the effect of the second term in Eq. (7) on the estimated velocity can be neglected. Taking into account that such a situation is rather frequent for measurements in the boundary layer, in the following we present the results corresponding to the case that the estimated power spectrum of the signal coincides with $W_{s}(k \Delta V, m \Delta \theta)$.

Based on the results of Ref. 41, the spectrum $W_{s}(k \Delta V, m \Delta \theta)$ can be written in the form

$$
\begin{aligned}
W_{s}(k \Delta V, m \Delta \theta)= & \frac{\Delta r}{\Delta V} \sum_{i=0}^{M^{\prime}-1} Q_{s}(\Delta r i) \\
& \times \operatorname{sinc}^{2}\left\{\pi\left[k-\frac{\left|V_{r}(\Delta r i, m \Delta \theta)\right|}{\Delta V}\right]\right\},
\end{aligned}
$$

where $\Delta r$ is the resolution along the optical axis $z^{\prime}$, $Q_{s}(\Delta r i)=\left\{\left(\pi k_{0} a_{0}^{2}\right)\left[\left(1-z^{\prime} / R\right)^{2}+(\Delta r i)^{2} /\left(k_{0} a_{0}^{2}\right)\right]\right\}^{-1}$ is the weighting function characterizing the contribution of each of $M^{\prime}$ path layers with the length $\Delta r$ to the normalized power of the signal $\left[\Delta r \sum_{i=0}^{M^{\prime}-1} Q_{s}(\Delta r i)=1\right] ; \quad \operatorname{sinc}(x)$ $=\sin (x) / x, k=0,1, \ldots, M_{D}-1 ; M_{D}$ is the number of spectral channels, $m=1,2, \ldots, M_{s}$; and $M_{s}$ is the number of the recorded spectra.

In accordance with Eq. (5), to estimate the velocity $V_{D}(m \Delta \theta)$ from the Doppler spectrum $W_{s}(k \Delta V, m \Delta \theta)$, we can use the following equation:

$V_{D}(m \Delta \theta)=\Delta V\left\{k_{\max }+\sum_{k=-k_{1}}^{k_{1}} k W_{s}\left[\left(k_{\max }+k\right) \Delta V, m \Delta \theta\right] / \hat{S}\right\}$,

where $k_{\max }$ is the number of the spectral channel corresponding to the peak in the spectral distribution; $\left[\Delta V\left(k_{\max }\right.\right.$ $\left.\left.-k_{1}\right), \Delta V\left(k_{\max }+k_{1}\right)\right]$ is the range of velocities, within which the value of $V_{D}$ is estimated; and $\hat{S}=\sum_{k=-k_{1}}^{k_{1}} W_{s}\left[\left(k_{\max }\right.\right.$ $+k) \Delta V, m \Delta \theta]$. It is obvious that the index $k_{1}$ introduced here must satisfy the following conditions:

$k_{1} \leqslant k_{\max }$

$k_{\max }+k_{1} \leqslant M_{D}-1$.

On substitution of Eq. (8) into Eq. (9), we pass on from summation over $k$ to integration with respect to the velocity $V(\Delta V k \rightarrow V)$ on the assumption that the chosen step $\Delta r$ of division along the optical axis $z^{\prime}$ satisfies the conditions $\Delta r \ll \Delta z$, where $\Delta z=(\lambda / 2)\left(R / a_{0}\right)^{2}$ is the effective longitudinal size of the sensed volume, ${ }^{42}$ and $M^{\prime} \Delta r>2 R$, we obtain

$$
\begin{aligned}
V_{D}(m \Delta \theta) & =\Delta r \sum_{i=0}^{M^{\prime}-1} Q_{s}(\Delta r i) V_{r}(\Delta r i, m \Delta \theta) \\
& =\int_{0}^{\infty} \mathrm{d} z^{\prime} Q_{s}\left(z^{\prime}\right) V_{r}\left(z^{\prime}, m \Delta \theta\right)
\end{aligned}
$$

Thus, the lidar estimate of the velocity [Eq. (9)] is the radial component of the wind velocity averaged over the sensed volume.

\subsection{Simulation Algorithm}

According to Eq. (8), to obtain Doppler spectra at different azimuthal angles $\theta=m \Delta \theta$, it is necessary to simulate random realizations of the radial velocity $V_{r}\left(z^{\prime}, \theta\right)$, which is related to the three components of the wind vector $\mathbf{V}(\mathbf{r}, t)$ by Eq. (6). Simulation of the 3-D random field for three velocity components with the required spatial resolution is impossible on ordinary up-to-date computers because of the limited memory and speed. Therefore, a simplified algorithm was used for simulation of the radial wind velocity.

Represent $V_{r}(\Delta r i, m \Delta \theta)$, with allowance for Eq. (10), in the form ${ }^{43}$

$V_{r}(\Delta r i, m \Delta \theta)=V_{D}(m \Delta \theta)+\Delta V_{r}(\Delta r i)$,

where 
$\Delta V_{r}(\Delta r i)=\widetilde{V}_{r}\left(R_{m}+\Delta r i\right)-\Delta r \sum_{i=0}^{M^{\prime}-1} Q_{s}(\Delta r i) \widetilde{V}_{r}\left(R_{m}+\Delta r i\right)$

$\tilde{V}_{r}=V_{r}-\left\langle V_{r}\right\rangle$ are fluctuations of the radial wind velocity, $R_{m}=\Delta r N_{m}, N_{m}=\{2 R \cos \varphi \sin [1 / 2(m-1) \Delta \theta] / \Delta r\}$. Fluctuations of the velocity $V_{D}$ are largely determined by turbulent eddies with the size $l>\Delta z$, while $\Delta V_{r}$ is determined by the small-scale turbulence $(l<\Delta z)$. This enables us to consider the random processes $V_{D}$ and $\widetilde{V}_{r}$ as independent and to simulate them separately. As follows from Eq. (12), based on the assumption of statistical homogeneity and isotropy of the atmospheric turbulence, we have gone from 2-D simulation of small scale fluctuations of radial velocity to 1-D realization of $\widetilde{V}_{r}$. Displacement of the focusing point $R_{m}$ along the scan circle in dependence on the azimuth angle $\theta$ in 2-D horizontal plane we have simulated by the displacement of $R_{m}$ on corresponding distance $\Delta r N_{m}$ along a 1-D realization of radial velocity.

The simulation of the random realizations of the radial velocity $\tilde{V}_{r}(i \Delta r)$ was performed in the spectral domain. ${ }^{32}$ The components of the spectrum of the unit complex (Gaussian) white noise are multiplied by the coefficients satisfying the spectral density of turbulent fluctuations of wind velocity in the atmosphere:

$S_{r}(\kappa)=\int_{-\infty}^{\infty} \mathrm{d} r\left\langle\tilde{V}_{r}(R+r) \tilde{V}_{r}(R)\right\rangle \exp (-2 \pi j \kappa r)$

Then the inverse Fourier transform is applied. The von Karman model is used ${ }^{44}$ as the spectrum $S_{r}(\kappa)$ :

$S_{r}(\kappa)=2 \sigma_{V}^{2} L_{V} /\left[1+\left(8.43 \kappa L_{V}\right)^{2}\right]^{5 / 6}$.

Here $L_{V}$ is the integral correlation scale of wind velocity (outer scale of turbulence). At the high frequencies $\kappa L_{V}$ $>1$, the spectral density $S_{r}(\kappa)$ should transform into the Kolmogorov-Obukhov spectrum ${ }^{45}$

$S_{r}(\kappa)=0.0375 C_{k} \varepsilon^{2 / 3} \kappa^{-5 / 3}$,

where $C_{k} \approx 2$ is the Kolmogorov constant, and $\varepsilon$ is the dissipation rate of the turbulent energy. Hence we can find that the parameters $\varepsilon, \sigma_{V}^{2}$, and $L_{V}$ should be related as

$\varepsilon=\frac{1.887}{C_{k}^{3 / 2}} \frac{\sigma_{V}^{3}}{L_{V}}$

The random process $V_{D}(m \Delta \theta)$ was simulated in the space of the azimuthal angles $\theta$ based on the correlation function $B_{D}\left(\theta_{m}, \theta_{l}\right)=\left\langle\widetilde{V}_{D}\left(\theta_{m}\right) \tilde{V}_{D}\left(\theta_{l}\right)\right\rangle$, where $\tilde{V}_{D}=V_{D}-\left\langle V_{D}\right\rangle$, derived on the assumption of the stationary, horizontally homogeneous, and isotropic wind velocity field and frozen turbulent inhomogeneities ${ }^{45}$ :

$$
\begin{aligned}
B_{D}\left(\theta_{m}, \theta_{l}\right)= & \int_{0}^{\infty} \int_{i=1}^{\infty} \mathrm{d} z^{\prime} \mathrm{d} z^{\prime \prime} Q_{s}\left(z^{\prime}\right) Q_{s}\left(z^{\prime \prime}\right) \sum_{k=1}^{3} \sum_{i}^{3}\left(\theta_{m}\right) S_{k}\left(\theta_{l}\right) \\
& \times B_{i k}\left[z^{\prime} \mathbf{S}\left(\theta_{m}\right)-z^{\prime \prime} \mathbf{S}\left(\theta_{l}\right)+\langle\mathbf{V}\rangle\left(\theta_{m}-\theta_{l}\right) / \omega_{0}\right],
\end{aligned}
$$

where $S_{1}=\sin \varphi ; \quad S_{2}=\cos \varphi \cos \theta ; \quad S_{3}=\cos \varphi \sin \theta ; \quad$ and $B_{i k}(\mathbf{r})=\left\langle\widetilde{V}_{i}\left(\mathbf{r}_{1}+\mathbf{r}\right) \widetilde{V}_{k}\left(\mathbf{r}_{1}\right)\right\rangle$ is the spatial correlation tensor of wind velocity fluctuations $\widetilde{V}_{1} \equiv \tilde{V}_{z}, \widetilde{V}_{2} \equiv \tilde{V}_{x}, \tilde{V}_{3} \equiv \tilde{V}_{y}$. In the case of the isotropic solenoidal wind velocity field, for $B_{i k}(\mathbf{r})$ we have ${ }^{46}$

$B_{i k}(\mathbf{r})=B_{V}(r) \delta_{i k}+\frac{r}{2} \frac{\mathrm{d} B_{V}(r)}{\mathrm{d} r}\left(\delta_{i k}-\frac{r_{i} r_{k}}{r^{2}}\right)$,

where $B_{V}(r)$ is the longitudinal correlation function of the wind velocity, $r=|\mathbf{r}|$, and $\delta_{i k}$ is the Kronecker delta. The correlation function $B_{V}(r)$ was calculated numerically

$B_{V}(r)=\frac{1}{2 \pi} \int \mathrm{d} \kappa S_{r}(\kappa) \exp (2 \pi j \kappa r)$,

with $S_{r}(\kappa)$ specified by Eq. (14).

Random realizations of $V_{D}(m \Delta \theta)$ were simulated by the method of linear transformation ${ }^{47}$

$V_{D}(m \Delta \theta)=\left\langle V_{D}(m \Delta \theta)\right\rangle+\sum_{m^{\prime}=1}^{m} a_{m m^{\prime}} \xi_{m^{\prime}}$,

where the elements of the matrix $a_{m m^{\prime}}$ are related to the elements of the correlation matrix $B_{D}(m \Delta \theta, l \Delta \theta)$ as

$B_{D}(m \Delta \theta, l \Delta \theta)=\sum_{m^{\prime}=1}^{m} a_{m m^{\prime}} a_{l m^{\prime}}$,

$\xi_{m}$ is a pseudorandom number distributed by the normal law with zero mean and unit variance, and $\left\langle\xi_{m} \xi_{m^{\prime}}\right\rangle=\delta_{m m^{\prime}}$.

\subsection{Simulation Results}

When simulating the Doppler spectra, we specified the following lidar parameters: wavelength $\lambda=10.6 \mu \mathrm{m}$ and initial radius of the sensing beam $a_{0}=7.5 \mathrm{~cm}$. Figure 2 shows the examples of simulation of the Doppler spectra $W_{s}(V)$ at $\left\langle V_{r}\right\rangle=10 \mathrm{~m} / \mathrm{s}$ and different values of $R, \sigma_{V}$, and $L_{V}$. We can see that an increase of $R$ or $\sigma_{V}$, as well as the decrease of $L_{V}$ leads to broadening of the spectrum. The dashed lines show the estimates of the velocity obtained using Eq. (9). The greater the asymmetry in the spectral distribution, the larger the deviation of this estimate from $\Delta V k_{\max }$.

After obtaining the velocity estimates at different azimuthal angles for one complete scanning cycle, the obtained values were fitted, in accordance with Eq. (6), by the least-squares method to the dependence 

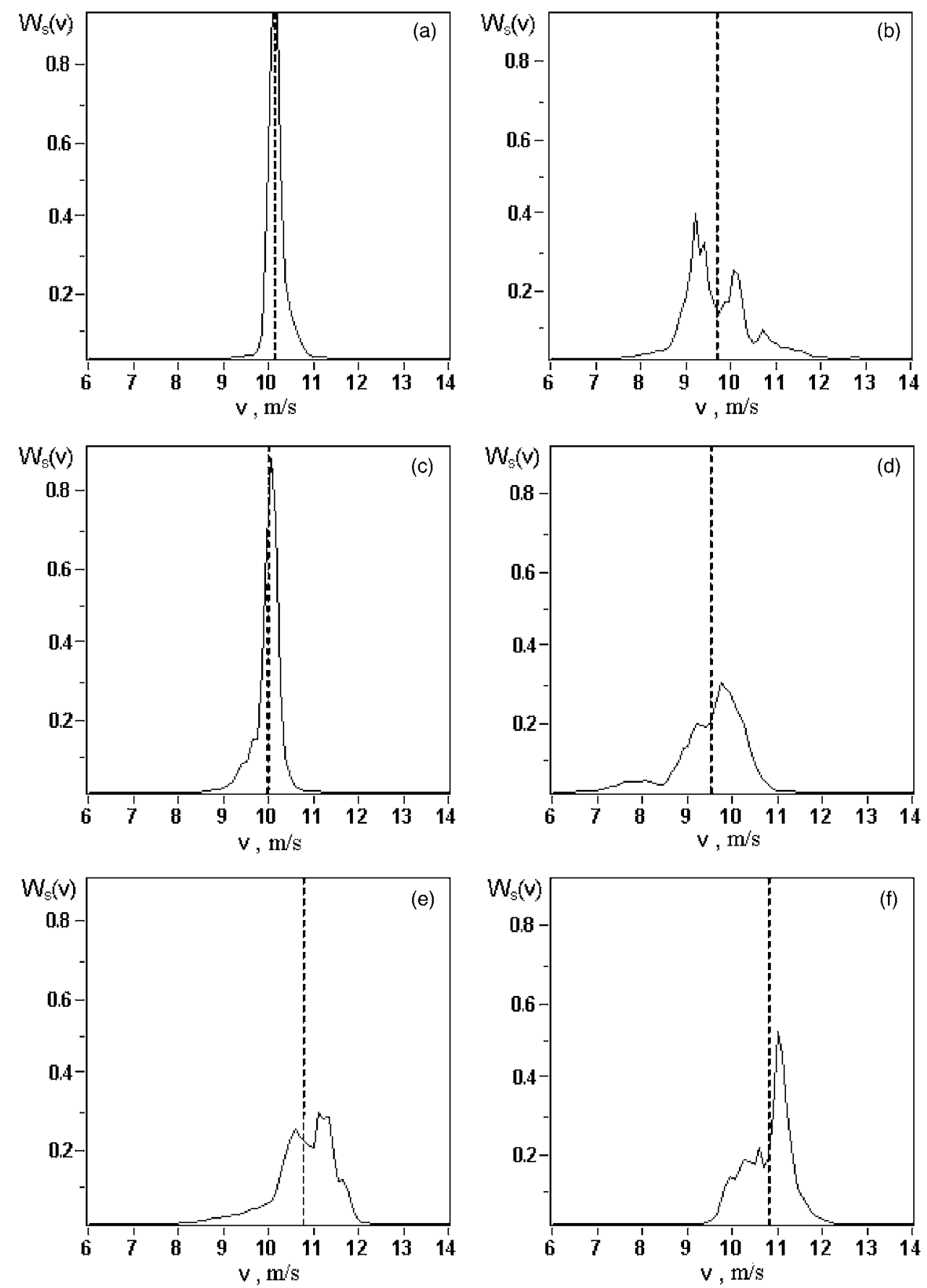

Fig. 2 Examples of simulation of Doppler spectra $W_{s}(V)$ at $\left\langle V_{r}\right\rangle=10 \mathrm{~m} / \mathrm{s}$ and (a) $\sigma_{V}=0.5 \mathrm{~m} / \mathrm{s}, L_{V}$ $=100$, and $R=100 \mathrm{~m}$; (b) $\sigma_{V}=1.5 \mathrm{~m} / \mathrm{s}, L_{V}=100$, and $R=100 \mathrm{~m}$; (c) $\sigma_{V}=1 \mathrm{~m} / \mathrm{s}, L_{V}=100$, and $R$ $=50 \mathrm{~m} ;(\mathrm{d}) \sigma_{V}=1 \mathrm{~m} / \mathrm{s}, L_{V}=100$, and $R=500 \mathrm{~m} ;(\mathrm{e}) \sigma_{V}=1 \mathrm{~m} / \mathrm{s}, L_{V}=30$, and $R=200 \mathrm{~m}$; and (f) $\sigma_{V}$ $=1 \mathrm{~m} / \mathrm{s}, L_{V}=300$, and $R=200 \mathrm{~m}$.

$\hat{V}_{r}(m \Delta \theta)=\sin \varphi \hat{V}_{z}+\cos \varphi\left[\cos (m \Delta \theta) \hat{V}_{x}+\sin (m \Delta \theta) \hat{V}_{y}\right]$,

from where the three components of the wind vector $\hat{\mathbf{V}}$ $=\left\{\hat{V}_{z}, \hat{V}_{x}, \hat{V}_{y}\right\}$ were estimated.

The simulation was carried out for the case when one complete turn of the beam around the vertical axis takes $T=7 \mathrm{~s}$ and the integration time of Doppler spectrum $t_{0}$
$=50 \mathrm{~ms}$. Then the number of the simulated spectra is $N_{\mathrm{sp}}$ $=T / t_{0}=140$, and the resolution in the azimuthal angle is $\Delta \theta=\omega_{0} t_{0}=2 \pi t_{0} / T \approx 2.57 \mathrm{deg}$. The spectra $W_{s}(V)$, whose peaks fell within the range $\Delta V k_{\max }<1 \mathrm{~m} / \mathrm{s}$, where rejected, and therefore the number of the obtained velocity estimates was $N_{V}<N_{\text {sp. }}$. It is obvious that the lower the mean wind velocity or the larger the angle $\varphi$, the smaller $N_{V}$. Figure 3 depicts the example of the dependence $V_{D}(m \Delta \theta)$ obtained 


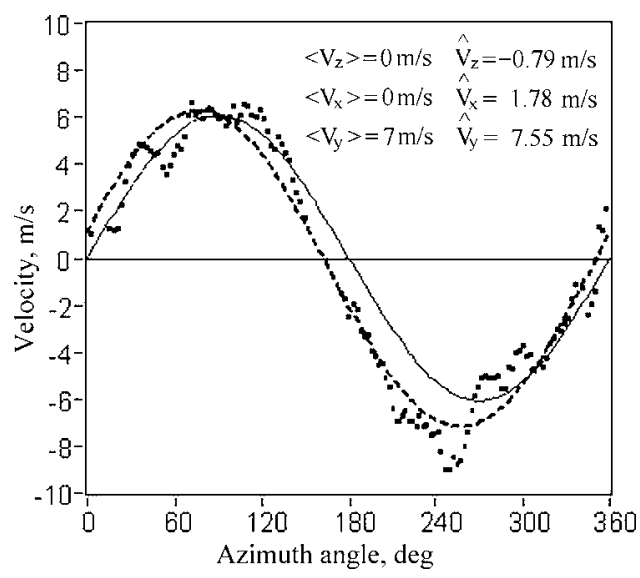

Fig. 3 Examples of estimations of $V_{D}(m \Delta \theta)$ (dots) and $V_{r}(m \Delta \theta)$ (dashed curve) from model Doppler spectra; the solid curve is $\left\langle\hat{V}_{r}(m \Delta \theta)\right\rangle$.

from simulated Doppler spectra. The dashed curve shows the dependence $\hat{V}_{r}(m \Delta \theta)$, while the solid curve stands for $\left\langle\hat{V}_{r}(m \Delta \theta)\right\rangle$. The components of the vectors $\langle\mathbf{V}\rangle$ and $\langle\hat{\mathbf{V}}\rangle$ are listed as well.

The data of numerical simulation for the different number of scans at the certain altitude $h$ were then used to determine the dissipation rate of turbulent energy $\varepsilon$, the variance of the wind velocity $\sigma_{V}^{2}$, and the outer scale of turbulence $L_{V}$. The methods for determination of these parameters from the data of measurements with a cw Doppler lidar are described elsewhere. ${ }^{15,42,48,49}$ Here, we only note that $\varepsilon$ is estimated from the spatial structure function of Doppler estimates of wind velocity obtained under different azimuth angles

$D[(m-n) \Delta \theta]=\left\langle\left[\widetilde{V}_{D}(m \Delta \theta)-\widetilde{V}_{D}(n \Delta \theta)\right]^{2}\right\rangle$,

where $\tilde{V}_{D}(m \Delta \theta)=V_{D}(m \Delta \theta)-\hat{V}_{r}(m \Delta \theta)$. To obtain the information concerning $\sigma_{V}^{2}$, it is necessary to additionally know the mean width of the Doppler spectra and the variance of deviations of an individual estimate of the wind velocity (over one scan) from that averaged over all scans. The outer

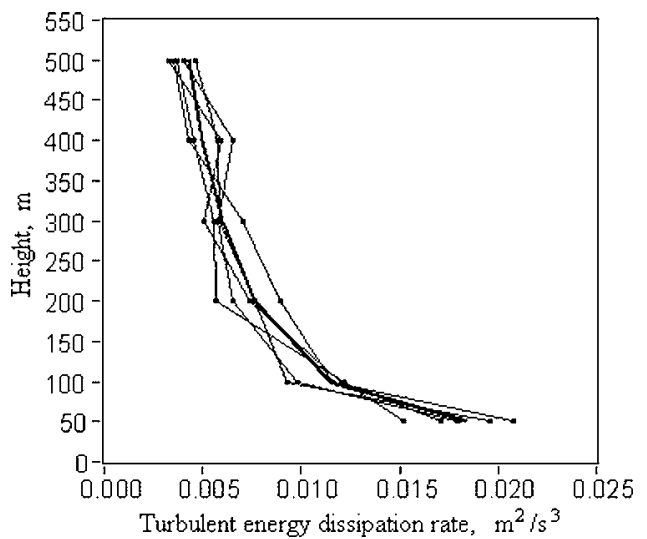

Fig. 4 Examples of retrieval of the vertical profiles of the turbulent energy dissipation rate from the model data for five scans (dots, connected by lines). The preset profile of the dissipation rate $\varepsilon(h)$ is shown by the bold curve.

scale of turbulence $L_{V}$ is calculated from the obtained estimates of $\varepsilon$ and $\sigma_{V}$ by Eq. (16).

In atmospheric measurements with the cw Doppler lidar to retrieve the vertical profiles of turbulence, ${ }^{15}$ we usually perform one or several (no more than three) continuous complete scans successively for each chosen altitude (the altitude was set by varying $R$ or $\varphi, h=R \sin \varphi$ ). Then the procedure was repeated many times with the certain time interval $T_{m} \sim 2$ to $5 \mathrm{~min}$. The time $T_{m}$ is enough for the turbulence to change significantly, and the obtained estimates of the turbulent parameters can be believed to be statistically independent. By analogy with the field experiment, for the fixed altitudes $h_{i}$, where $i=1,2, \ldots, 6$, we simulated independent arrays of the Doppler spectra. The angle $\varphi=30 \mathrm{deg}$ was kept the same for all the altitudes. Each of the arrays corresponded to the set of the spectra obtained during individual complete scan.

The parameters to be retrieved were the vertical profiles of the mean wind velocity $U(h)$, the dissipation rate of turbulent energy $\varepsilon(h)$, the variance of the wind velocity $\sigma_{V}^{2}(h)$, and the outer scale of turbulence $L_{V}(h)$ calculated in accordance with the models ${ }^{50}$ (see Table 1).

Figure 4 exemplifies the vertical profiles of the dissipa-

Table 1 Values of mean wind velocity, turbulent energy dissipation rate, wind velocity variance, and outer scale of turbulence.

\begin{tabular}{llcccl}
\hline \hline & $h(\mathrm{~m})$ & $U(\mathrm{~m} / \mathrm{s})$ & $\sigma_{V}(\mathrm{~m} / \mathrm{s})$ & $L_{V}(\mathrm{~m})$ & $\varepsilon\left(\mathrm{m}^{2} / \mathrm{s}^{3}\right)$ \\
\hline 1 & 50 & 6.90 & 1.23 & 133 & 0.018 \\
2 & 100 & 7.77 & 1.20 & 200 & 0.011 \\
3 & 200 & 8.63 & 1.15 & 266 & 0.0077 \\
4 & 300 & 9.14 & 1.11 & 300 & 0.0060 \\
5 & 400 & 9.50 & 1.07 & 320 & 0.0050 \\
6 & 500 & 9.78 & 1.02 & 333 & 0.0043 \\
\hline \hline
\end{tabular}


tion rate of turbulent energy as retrieved from the data of numerical simulation with five scans at every level $h_{i}$. The bold curve is the profile $\varepsilon\left(h_{i}\right)$ presented in Table 1 . We can see that the estimates of the dissipation rate $\hat{\varepsilon}\left(h_{i}\right)$ are concentrated near the initial profile $\varepsilon\left(h_{i}\right)$, that is, a pronounced regular shift of the estimates $\hat{\varepsilon}\left(h_{i}\right)$ is absent. The bias of the dissipation rate estimate $s_{\varepsilon}=\langle\hat{\varepsilon}-\varepsilon\rangle$ we calculated using $N$ $\times 1000$ independent estimates $\hat{\varepsilon}$ obtained from the data of numerical simulation, where $N$ is the number of scans. From the obtained results, it follows that for the lowest height level, the relative bias $s_{\varepsilon} / \varepsilon$ is negligible, less than $0.8 \%$. The bias $s_{\varepsilon} / \varepsilon$ varies nonregularly with height, taking values between 2 and $5 \%$ for the height range from 100 to $500 \mathrm{~m}$.

To calculate the relative error in estimation of the dissipation rate of turbulent energy $\sigma_{\varepsilon}=\left[\left\langle(\hat{\varepsilon}-\varepsilon)^{2}\right\rangle\right]^{1 / 2} / \varepsilon$, we used the same $N \times 1000$ independent estimates $\hat{\varepsilon}$ for each height level, which were used in the calculations of $s_{\varepsilon}$. Figure 5(a) shows the dependence of the relative error $\sigma_{\varepsilon}$ on the number of scans for the altitudes $h_{i}$ presented in Table 1 . We can see that the values of $\sigma_{\varepsilon}$ for different altitudes differ insignificantly, and with an increase of $N$ (the amount of processed data) they decrease down to the level $\sim 18 \%$ for $N=10$.

Figure 5(b) shows the calculated relative error in estimation of the variance of wind velocity $\sigma_{\sigma_{V}^{2}}=\left[\left\langle\left(\hat{\sigma}_{V}^{2}\right.\right.\right.$ $\left.\left.\left.-\sigma_{V}^{2}\right)^{2}\right\rangle\right]^{1 / 2} / \sigma_{V}^{2}$. The curves are numbered in accordance with the numbers of the sensing altitudes $h_{i}$ in Table 1 . In contrast to $\sigma_{\varepsilon}$, the values of the error $\sigma_{\sigma_{V}^{2}}^{2}$ turn out to be different at different altitudes. The lower the altitude $h_{i}$, the greater the relative error in estimation of the variance of wind velocity. Such a behavior of $\sigma_{\sigma_{V}^{2}}$ is quite understandable if we take into account the fact that one of the terms in the equation for estimation of the variance $\hat{\sigma}_{Y}^{2}$ is the mean square of the width of the Doppler spectrum. ${ }^{15}$ The higher the altitude $h$ at the fixed angle $\varphi$, the larger the longitudinal size of the sensed volume $\Delta z$ and, consequently, the larger are turbulent vortices contributing to broadening of the Doppler spectrum, so that this spectrum becomes more informative with respect to random variations of the wind velocity in the sensed volume [compare Figs. 2(c) and 2(d)]. From Fig. 5(b) it follows that the value of $\sigma_{\sigma_{V}^{2}}$ ranges within 17 to $25 \%$ for the number of scans $N=10$.

The values of $\hat{\sigma}_{V}$ and $\hat{\varepsilon}$ enable us to estimate the outer scale of turbulence $L_{V}$ by Eq. (16). Figure 5(c) depicts the relative error of estimation of the outer scale of turbulence $\sigma_{L_{V}}=\left[\left\langle\left(\hat{L}_{V}-L_{V}\right)^{2}\right\rangle\right]^{1 / 2} / L_{V}$ at different altitudes $h_{i}$. The curves are numbered in accordance with Fig. 5(b). It is obvious that the error of the estimate $\hat{L}_{V}$ is caused by random variations of $\hat{\sigma}_{V}$ and $\hat{\varepsilon}$. Therefore, the error $\sigma_{L_{V}}$ exceeds the corresponding values of $\sigma_{\varepsilon}$ and $\sigma_{\sigma_{V}^{2}}$. For $N=10$, the value of $\sigma_{L_{V}}$ ranges within $\sim 20$ to $40 \%$.

The relative biases of the estimates of $\sigma_{V}^{2}$ and $L_{V}$ for different heights lie in the range between 0.7 and $4.3 \%$ and 1.5 and $6.2 \%$, correspondingly.

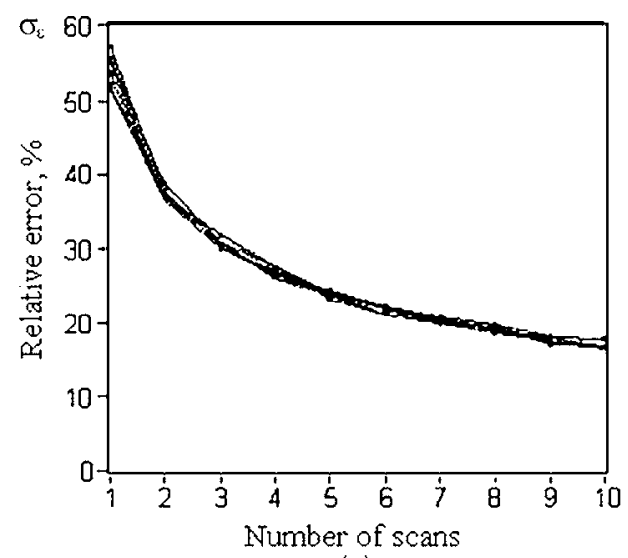

(a)

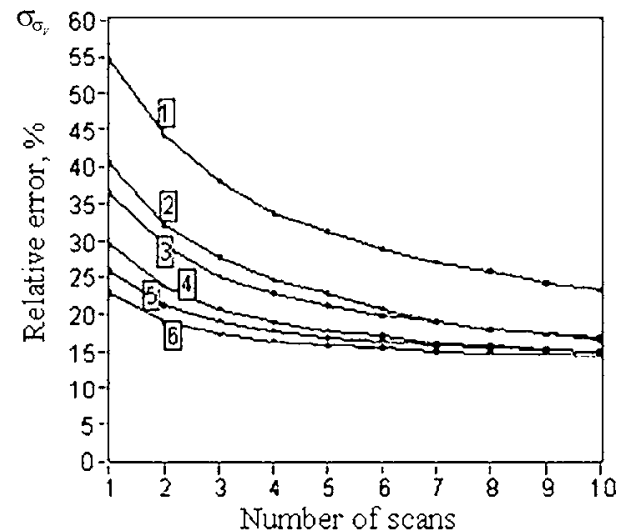

(b)

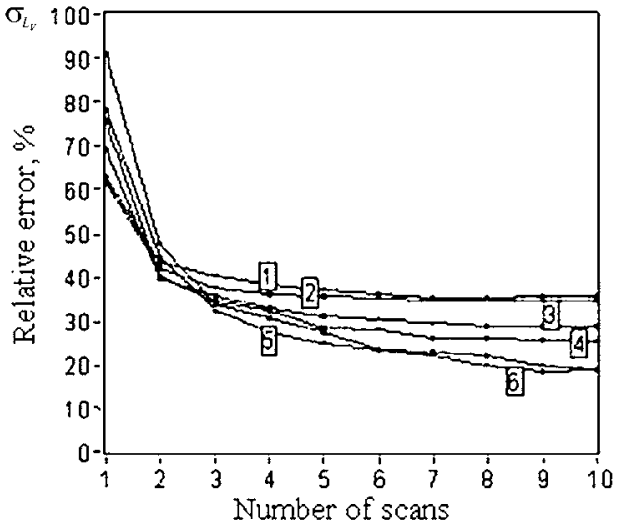

(c)

Fig. 5 Relative error in estimation of (a) the dissipation rate of turbulent energy, (b) variance of the wind velocity, and (c) outer scale of turbulence as a function of the number of scans. The curves are numbered in accordance with the heights presented in Table 1.

\section{Retrieval of Vertical Wind Profiles from Measurements with a Space-Borne Scanning Pulsed CDL}

The current $j_{s}$ of a pulsed CDL at the time $t$ can be presented in the form ${ }^{27,28,51}$

$j_{s}(t)=\operatorname{Re}[y(t)]$

where 

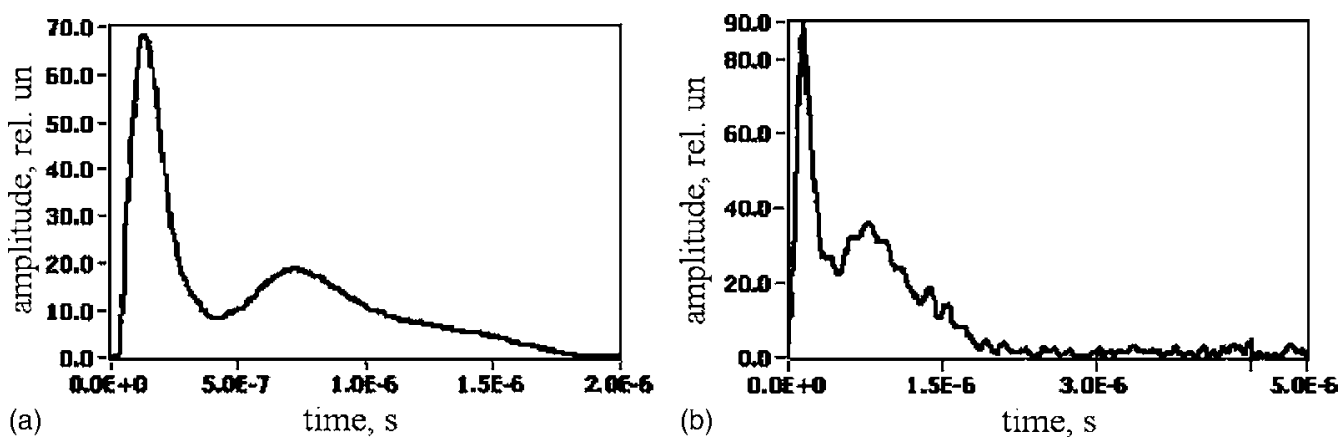

Fig. 6 (a) Shape of the WIND lidar pulse as a result of averaging over 5500 pulses and (b) example of a pulse of the $\mathrm{CO}_{2}$ laser employed in the WIND lidar system.

$$
\begin{aligned}
y(t)= & 2 \frac{\mathrm{e} \eta_{Q}}{h \nu} \frac{K(R)}{R} P_{L}^{1 / 2} \sum_{l=1}^{n_{s}} P_{T}^{1 / 2}\left(t-2 z_{l} / c\right) \alpha_{l} \\
& \times Q\left(\rho_{l}\right) \exp \left\{2 j k z_{l}+2 \pi j\left[\Delta f-\frac{2}{\lambda} V_{r}\left(z_{l}\right)\right] t\right\}, \\
Q\left(\rho_{l}\right)= & R \lambda \int_{-\infty}^{\infty} \mathrm{d}^{2} \rho W(\rho) A_{L}^{*}(\rho) \int_{-\infty}^{\infty} \mathrm{d}^{2} \rho^{\prime} A_{T}\left(\rho^{\prime}\right) W\left(\rho^{\prime}\right) \\
& \times G\left(\rho_{l}, R ; \rho^{\prime}, 0\right) G\left(\rho_{l}, R ; \rho, 0\right),
\end{aligned}
$$

where $R=c t / 2$ is the distance to the sensed volume; $c$ is the speed of light; $K(R)=\exp \left[-\int_{0}^{R} \mathrm{~d} z^{\prime} \alpha_{a}\left(z^{\prime}\right)\right] ; \alpha_{a}$ is the extinction coefficient of laser radiation in the atmosphere; $A_{L}(\rho)$ $=E_{L}(\rho) / P_{L}^{1 / 2}$ and $A_{T}(\rho)=E_{T}(\rho, t) / P_{T}^{1 / 2}(t)$ are normalized amplitudes of the local oscillator and sensing pulses $P_{T}(t)$ $=\int_{-\infty}^{\infty} \mathrm{d}^{2} \rho\left|E_{T}(\rho, t)\right|^{2} \quad$ and $\quad P_{L}=\int_{-\infty}^{\infty} \mathrm{d}^{2} \rho\left|E_{L}(\rho)\right|^{2} ; \quad U_{P}$ $=\int_{-\infty}^{\infty} \mathrm{d} t P_{T}(t)$ is energy of the sensing pulse; $W(\rho)$ is the pupil function of the transceiving telescope; $G$ is the Green's function of the parabolic equations describing optical wave propagation to the sensed volume and return, ${ }^{52}$ $\Delta f$ is the intermediate frequency; and $V_{r}(z)$ is the radial component of the wind velocity at the distance $z$ from the lidar. The rest parameters are described in Eqs. (1) and (2).

Pulsed CDLs widely employ laser sources of two types: solid-state lasers operating at the wavelength of $2 \mu \mathrm{m}$ and $\mathrm{CO}_{2}$ lasers operating at the wavelength $10.6 \mu \mathrm{m}$. In the first case, the sensing pulse is well described by the Gaussian distribution

$P_{T}(t)=\left(U_{P} / \sqrt{\pi \sigma}\right) \exp \left(-t^{2} / \sigma^{2}\right)$

where $\sigma=t_{p}$ is the pulse duration determined by the equation $P_{T}\left(t_{P}\right) / P_{T}(0)=e^{-1}$. In the second case, it is specified in the form shown in Fig. 6(a). This form is the result of averaging of a series of pulses generated ${ }^{8,9}$ by the WIND $\mathrm{CO}_{2} \mathrm{CDL}$ during the experiments.

For the mean power of the valid component of the photocurrent $S=(1 / 2) \overline{y(t) y^{*}(t)}$ from Eq. (23) we have ${ }^{27}$

$S=2\left[e \eta_{Q} /(h \nu)\right]^{2} P_{S} P_{L} \eta_{H}$,

where $P_{S}=A_{R} \beta_{\pi}(R) K^{2}(R) c U_{P} /\left(2 R^{2}\right)$ is the power of the received signal in the case of incoherent detection, $A_{R}$ $=\int_{-\infty}^{\infty} \mathrm{d}^{2} \rho W^{2}(\rho)$ is the aperture area of the transceiving tele- scope, and $\eta_{H}=A_{R}^{-1} \int_{-\infty}^{\infty} \mathrm{d}^{2} \rho_{l} \overline{\left|Q\left(\rho_{l}\right)\right|^{2}}$ is the heterodyning efficiency. Along with the component $y(t)$, the lidar return contains the noise component $n(t)$. For a Doppler lidar operating under ideal conditions when the main source of noise is the local oscillator shot noise described by the Poisson statistics and there is no atmospheric refractive turbulence, for the mean power of noise $N=\overline{|n|^{2}}$, we have ${ }^{53}$

$N=2 e^{2} \eta_{Q} P_{L} B /(h \nu)$

where $B$ is the detector bandwidth. Then the equation for the signal-to-noise ratio $\mathrm{SNR}=\mathrm{S} / \mathrm{N}$ can be written as

$\mathrm{SNR}=\frac{\eta_{Q} \eta_{H} P_{S}}{h \nu B}$

The SNR value is the mean number of photoelectrons detected $^{29}$ coherently for the time $\sim B^{-1}$.

Doppler lidar data is well approximated ${ }^{29}$ as a zeromean complex Gaussian random vector $\mathbf{Z}$ with elements

$Z\left(m T_{S}\right)=\frac{1}{\sqrt{2}} y\left(t+m T_{S}\right)+n\left(m T_{S}\right)$

where $T_{S}=B^{-1}$ is the time of reading the complex signal, $m=0,1, \ldots, M-1$, and $M T_{s}$ defines the total observation time per Doppler frequency estimate. The signal $Z\left(m T_{S}\right)$ satisfies the following equations: $\overline{Z\left(m T_{S}\right)}=\overline{Z\left(m T_{S}\right) Z\left(l T_{S}\right)}$ $=0$ and $\overline{Z\left(m T_{S}\right) Z^{*}\left(l T_{S}\right)}=S K_{y}\left(m T_{S}, l T_{S}\right)+N \delta_{m l}$, where $K_{y}$ is the correlation coefficient of the complex value of the signal valid component obtained through averaging of the product $y\left(t+m T_{S}\right) y^{*}\left(t+l T_{S}\right)$ over all fluctuating parameters in Eq. (23), except for the wind velocity; and $\delta_{m l}$ is the Kronecker delta $\left(\delta_{m l, m=l}=1, \delta_{m l, m \neq l}=0\right)$.

The Doppler frequency $f_{D}$ can be selected from the measured series $Z\left(m T_{S}\right)$ only within the Nyquist interval $\left[0,1 / T_{S}\right]$. Having passed on from $Z\left(m T_{S}\right)$ to $Z\left(m T_{S}\right)$ $\times \exp \left(-2 \pi j \Delta f m T_{S}\right) / \sqrt{N}$ and assumed $\Delta f=1 /\left(2 T_{S}\right)$ with allowance for the Doppler relation $V_{D}(R)=(\lambda / 2) f_{D}$, we obtain that the estimate of the radial wind velocity $V_{D}(R)$ falls within the interval $\left[-\lambda /\left(4 T_{S}\right), \lambda /\left(4 T_{S}\right)\right]$. Then from Eqs. (23)-(28) for the correlation function of the complex signal $B_{z}\left(m T_{S}, l T_{S}\right)=\overline{Z\left(m T_{S}\right) Z^{*}\left(l T_{S}\right)}$ we have the equation 


$$
\begin{aligned}
B_{z}\left(m T_{S}, l T_{S}\right)= & \operatorname{SNR} \frac{2}{c U_{P}} \int_{-\infty}^{\infty} \mathrm{d} z^{\prime} P_{T}^{1 / 2}\left(m T_{S}-2 z^{\prime} / c\right) P_{T}^{1 / 2}\left(l T_{S}\right. \\
& \left.-2 z^{\prime} / c\right) \exp \left[j \frac{4 \pi}{\lambda}(l-m) T_{S} V_{r}\left(R+z^{\prime}\right)\right]+\delta_{m l}
\end{aligned}
$$

One of the methods to obtain the Doppler estimate of the radial velocity $V_{D}$ is the argument correlation function (ACF) method, ${ }^{32}$ in which $V_{D}$ is estimated by the argument of the correlation function

$V_{D}(R)=\lambda \arg \left[\hat{B}_{z}\left(T_{s}\right)\right] /\left(4 \pi T_{s}\right)$,

where $\hat{B}_{z}\left(T_{s}\right)=(1 / M-1) \sum_{i=0}^{M-2} Z\left(i T_{s}\right) Z^{*}\left[(i+1) T_{s}\right]$ is the unbiased estimate of the correlation function of the signal [Eq. (29)] with the time shift $T_{S}$.

Numerical simulation of pulsed CDL signal $Z\left(m T_{S}\right)$ is carried out in the following way. ${ }^{15,32,51}$ The area occupied in space by the pulse $P_{T}(t)$ along the direction of propagation is divided into $n_{L}$ layers, and the signal recorded at the time $m T_{S}$ is a sum of the contributions from all these layers and noise:

$$
\begin{aligned}
Z\left(m T_{S}\right)= & {\left[\frac{\mathrm{SNR}}{2 \sum_{k=0}^{n_{L}} P_{T}(\Delta p k)}\right]^{1 / 2} \sum_{k=0}^{n_{L}} a(k+m) P_{T}^{1 / 2}(\Delta p k) } \\
& \times \exp \left\{-j \frac{4 \pi}{\lambda} m T_{S} V_{r}[\Delta p(k+m)]\right\}+\frac{1}{\sqrt{2}} n_{m},
\end{aligned}
$$

where $\Delta p$ is the layer thickness, $a(k)$ and $n_{m}$ are independent complex random numbers with the real and imaginary parts distributed by the Gaussian law with zero mean and unit variance, and $V_{r}$ are real random values of wind velocity. The number of layers $n_{L}$ and layer thickness $\Delta p$ should provide reproducing pulse volume with good resolution. ${ }^{32}$ The spectral method of simulation of $V_{r}(\Delta p k)$ is described in Sec. 2.1.

The accuracy of CDL velocity estimates for a spacebased platform is studied in Refs. 34 and 35. There the effectiveness of accumulating the lidar signal over multiple lidar shots is considered to estimate the radial velocity averaged over measurement cell. Both the random variability of the wind field and aerosol backscatter as well as wind shear are included in the analysis in Ref. 34. Here, we discuss the algorithm for determining the horizontal wind velocity from space-borne scanning CDL signal directly without preliminary estimation of the radial velocity.

The algorithm for retrieval of the wind speed and direction from measurements with a space-borne scanning CDL is analyzed based on simulation with the virtual Doppler lidar instrument information-expert computer system. ${ }^{54}$ The virtual instrument system consists of several modules and enables simulation of the return of a space- or airborne scanning Doppler lidar. The first module contains the initial information about the lidar parameters, sensing geometry, and the atmosphere. The system uses the real data of the German Weather Service (GWS) on the global distribution of atmospheric parameters for the period of January 19 to
30, 1998, which enables us to study the capabilities of lidar wind sensing in the atmosphere under conditions that are maximally close to real conditions. The second module serves to simulate the lidar sensing process. It outputs realizations of the photocurrent arising in the lidar receiving circuit. The third module processes the simulated data to retrieve the vertical profiles of the radial wind component with a preset height resolution. Then the calculated vertical profiles of the wind speed and direction are accumulated in the output module and can be compared with the initial GWS wind data. The results of such a comparison enable us to judge the efficiency of lidar measurements of wind fields.

\subsection{Simulation of the Turbulent Wind Field in the Troposphere}

In the atmospheric module of the virtual instrument system, the GWS data on the atmospheric parameters all over the globe are set on the grid with the spatial resolution of 1.125 deg longitude $\times 1.121$ deg latitude. For simulation of the wind turbulence, the data on the zonal $V_{z}\left(z_{i}\right)$ and meridional $V_{m}\left(z_{i}\right)$ components of the mean wind velocity (in meters per second), temperature $T\left(z_{i}\right)$ (in kelvins), and the turbulent viscosity $K_{m}\left(z_{i}\right)$ and turbulent heat exchange $K_{h}\left(z_{i}\right)$ coefficients (in square meters per second) are used. Here $z_{i}$ is the altitude from 0 to $30 \mathrm{~km}$, for which the values of the meteorological parameters are specified, $i=1,2, \ldots$, 20.

We assume that random wind fields are isotropic with the parameters depending on the height. This assumption is based on the numerous experimental data, ${ }^{44}$ where the Karman model is used in analysis of the tropospheric wind spatial statistics under a condition of neutral thermal stratification. Based of this assumption, the procedure of simulation of random realizations of the radial velocity is divided into two stages:

1. calculation of the vertical profiles of the variance of wind velocity fluctuations $\sigma_{V}^{2}(z)$ and the integral scale of turbulence $L_{V}(z)$ from the GWS data

2. simulation of random realizations of the 2-D wind field in the plane of propagation of the sensing pulse with the use of the von Karman model of the spatial spectrum of turbulence inhomogeneities

In the atmospheric surface layer, the parameters $\sigma_{V}$ and $L_{V}$ are calculated using the equation of balance of turbulent energy. In this case, in accordance with the turbulent kinetic energy (TKE) parameterization of the boundary layer, ${ }^{55,56}$ for the dissipation rate of turbulent energy at the altitude $z_{i}$ we have

$$
\begin{aligned}
\varepsilon\left(z_{i}\right)= & K_{m}\left(z_{i}\right)\left\{\left[\frac{\mathrm{d} V_{z}}{\mathrm{~d} z}\left(z_{i}\right)\right]^{2}+\left[\frac{\mathrm{d} V_{m}}{\mathrm{~d} z}\left(z_{i}\right)\right]^{2}\right\} \\
& -\frac{g}{\theta\left(z_{i}\right)} K_{h}\left(z_{i}\right) \frac{\mathrm{d} \theta}{\mathrm{d} z}\left(z_{i}\right),
\end{aligned}
$$

where $\theta\left(z_{i}\right)$ is the potential temperature calculated from $T\left(z_{i}\right)$ through the adiabatic gradient, ${ }^{57} \mathrm{~g}\left[\mathrm{~m}^{2} / \mathrm{s}\right]$ is the freefall acceleration. According to theory of turbulence (see, for 
example, Refs. 44-46 and 58), the turbulent energy can be calculated by the following equation:

$E\left(z_{i}\right)=C_{E}\left[\varepsilon\left(z_{i}\right) K_{m}\left(z_{i}\right)\right]^{1 / 2}$,

where $C_{E}=5.73$ is an empirical constant. Whence, by the equation

$\sigma_{V}\left(z_{i}\right)=C_{V}\left[E\left(z_{i}\right)\right]^{1 / 2}$,

where $C_{V}=1.04$, the variance (standard deviation) of fluctuations of the longitudinal wind velocity component $\sigma_{V}\left(z_{i}\right)$ was calculated. The values of $L_{V}\left(z_{i}\right)$ were determined from calculated $\varepsilon\left(z_{i}\right)$ and $\sigma_{V}\left(z_{i}\right)$ by Eq. (16).

In the surface layer, the turbulent energy $E$ (and $\sigma_{V}$ ) is known to vary insignificantly with height, while the outer scale increases proportionally to the height. Therefore, for the surface layer the following approximate equations were used to estimate the sought parameters:

$\sigma_{s}=\left[\frac{1}{n} \sum_{i=1}^{n} \sigma_{V}^{2}\left(z_{i}\right)\right]^{1 / 2}$

and

$L_{s}=C_{s} z$,

where $C_{s}=(1 / n) \sum_{i=1}^{n}\left[L_{V}\left(z_{i}\right) / z_{i}\right], \sigma_{s}^{2}$ is the variance, and $L_{s}$ is the correlation scale for the surface layer $z_{i} \in\left[0, h_{s}\right]$, and $h_{s}$ is the height of the surface layer. To estimate $\sigma_{s}$ and $L_{s}$, we used the GWS data for the three lowest height levels.

Friction, which significantly influences airflow near the earth's surface, weakens with the increase of the height in the boundary layer and for the heights $z \gg h_{B}$, where $h_{B}$ is the height of the atmospheric boundary layer, friction can be neglected. In the free atmosphere at the heights $z \gg h_{B}$, the turbulent energy is generated by other sources, and, as was shown by the experimental data, ${ }^{44}$ the turbulence intensity $\sigma_{V}(z) / U(z)$, where $U(z)=\left[V_{z}^{2}(z)+V_{m}^{2}(z)\right]^{1 / 2}$, is about 0.05 for wind velocity fluctuations with the spatial scales not exceeding $10 \mathrm{~km}$. The parametric fitting of the measured spatial spectra of the turbulent wind field to the von Karman model [Eq. (14)] yields the estimate of $500 \mathrm{~m}$ for the integral correlation scale of velocity in the free atmosphere. ${ }^{44}$ Based on these data, for estimation of the vertical profiles of $\sigma_{V}^{2}$ and $L_{V}$ we used the following simple model:

$\sigma_{V}^{2}(z)=\sigma_{s}^{2} \exp \left[-2\left(z / h_{B}\right)\right]+\sigma_{f}^{2}\left\{1-\exp \left[-2\left(z / h_{B}\right)\right]\right\}$,

$L_{V}(z)=\frac{C_{s} z}{1+\left(C_{s} z / L_{f}\right)}$,

where $\sigma_{f}^{2}=[\gamma U(z)]^{2}$ is the variance; $L_{f}$ is the integral correlation scale of wind velocity in the free atmosphere, $\gamma$ $=0.05$, and $L_{f}=500 \mathrm{~m}$. The height of the boundary layer $h_{B}$ depends on the thermal stratification in the atmosphere and was estimated based on the GWS data.

To simulate random realizations of the radial velocity, we used the spectral method [see Sec. 2.1, Eq. (13)]. As used in simulation the 1-D spatial spectra of the longitudi- nal $S_{u}\left(z, \kappa_{z}\right)$ and lateral $S_{u}\left(z, \kappa_{x}\right)$ components of the wind velocity and the 2-D spatial spectrum $S_{u}\left(z, \kappa_{z}, \kappa_{x}\right)$, according to the von Karman model [Eq. (14)] are written as

$$
\begin{aligned}
S_{u}\left(z, \kappa_{z}\right)= & \frac{2 \sigma_{V}^{2}(z) L_{V}(z)}{\left\{1+\left[8.43 L_{V}(z) \kappa_{z}\right]^{2}\right\}^{5 / 6}}, \\
S_{u}\left(z, \kappa_{x}\right)= & \frac{\sigma_{V}^{2}(z) L_{V}(z)}{\left\{1+\left[8.43 L_{V}(z) \kappa_{x}\right]^{2}\right\}^{5 / 6}} \\
\times & \left\{1+\frac{5}{3} \frac{\left[8.43 L_{V}(z) \kappa_{x}\right]^{2}}{1+\left[8.43 L_{V}(z) \kappa_{x}\right]^{2}}\right\}, \\
S_{u}\left(z, \kappa_{z}, \kappa_{x}\right)= & \frac{1}{6 \pi} \frac{\sigma_{V}^{2}(z)\left(8.43 L_{V}(z)\right)^{2}}{\left\{1+\left[8.43 L_{V}(z)\right]^{2}\left(\kappa_{z}^{2}+\kappa_{x}^{2}\right)\right\}^{4 / 3}} \\
& \times\left\{1+\frac{8}{3} \frac{\left[8.43 L_{V}(z) \kappa_{x}\right]^{2}}{1+\left[8.43 L_{V}(z)\right]^{2}\left(\kappa_{z}^{2}+\kappa_{x}^{2}\right)}\right\},
\end{aligned}
$$

where the parameters $\sigma_{V}^{2}(z)$ and $L_{V}(z)$ are calculated by Eqs. (37) and (38).

The vertically (along the $z$ axis) inhomogeneous random wind field $\tilde{V}(z)$ was simulated by the following scheme.

First, the array of the values $\tilde{V}_{0}\left(z_{k}\right)$, where $z_{k}=z_{0}+\Delta z k, k$ $=0,1,2, \ldots, N_{z}$, was simulated for the constant values of $\sigma_{V}^{2}(z)=\sigma_{V_{0}}^{2}$ and $L_{V}(z)=L_{V_{0}}$. Then the obtained array was corrected:

$\tilde{V}\left(z_{k}\right)=\left[\sigma_{V}\left(z_{k}\right) / \sigma_{V_{0}}\right] \widetilde{V}_{0}\left(\hat{z}_{k}\right)$,

where

$\hat{z}_{k}=\left(z_{0}+\Delta z k\right) \frac{L_{V}\left(z_{k}\right)}{L_{V_{0}}}$,

$\sigma_{V_{0}}=\sigma_{V}\left(z_{0}\right), L_{V_{0}}=L_{V}\left(z_{0}\right)$, and $z_{0}$ is the initial height.

\subsection{Simulation of Retrieval of Wind Profiles from the Array of Estimates of the Radial Velocity}

Figure 7 depicts the schematic layout of sensing by a scanning Doppler lidar installed on a satellite. The arrow indicates the satellite direction. The dots show the distribution of single sensing pulses in the horizontal plane. The size of the grid cells corresponds to the latitude and longitude resolution of the GWS data. The information about the vertical profiles of the wind speed and direction is retrieved from sensing pulses falling within one such cell using the relation between the radial velocities, propagation directions (nadir, azimuthal scanning angle), and the wind velocity vector (two horizontal components; the vertical one is assumed zero) on the assumption that the wind is homogeneous within the cell at the chosen height.

In numerical simulation, we set the following parameters: satellite height of $400 \mathrm{~km}$; nadir of $35 \mathrm{deg}$; one complete scan for $10 \mathrm{~s}$, pulse repetition frequency of sensing pulses of $10 \mathrm{~s}^{-1}$, pulse duration of $0.1 \mu \mathrm{s}$, pulse energy of $0.5 \mathrm{~J}$, wavelength of $2.02184 \mu \mathrm{m}$, telescope diameter of 70 $\mathrm{cm}$, data readout rate of $200 \mathrm{MHz}$, bandwidth of $50 \mathrm{MHz}$ 


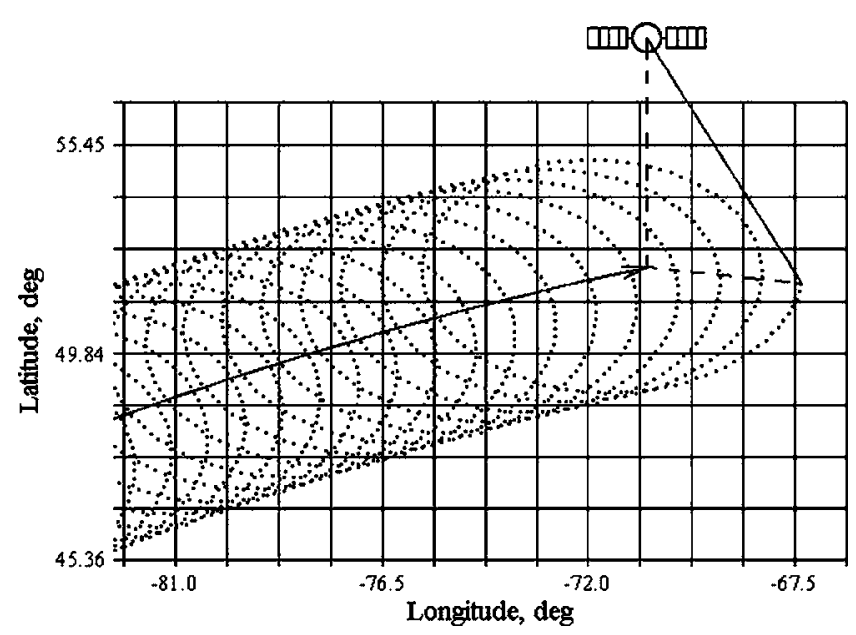

Fig. 7 Geometry of sensing with a space-borne scanning Doppler lidar.

for the processed data, vertical resolution of $1258 \mathrm{~m}$, and horizontal resolution of 1.125 deg longitude $\times 1.121$ deg latitude.

Figure 8 shows examples of the vertical profiles of wind speed and direction as retrieved from the model lidar data with and without regard for noise. The wind speed and direction were calculated from the estimates of the radial wind velocity for the sensing pulses falling within the selected cell of the GWS data. The radial velocity was estimated from the peak position in the spectral distribution of the power spectra of model lidar data. The dashed curves show the GWS data. We can see that in the absence of noise, the wind velocity is retrieved with high accuracy. However, in the presence of noise, because of the low SNR, the valid component of the spectrum is lost in noise peaks, and the error in retrieval of the wind velocity is very high.

At the low SNR, it is quite probable that a noise peak exceeds the peak of the Doppler spectrum, which leads to an incorrect estimation of the frequency assigned to the radial wind velocity. The obtained false estimate of the Doppler frequency has a uniform distribution in the 50$\mathrm{MHz}$ band, or, with allowance for the Doppler relation $V$ $=(\lambda / 2) f$, in the velocity range of $\pm 50 \mathrm{~m} / \mathrm{s}$ approximately.

A possible way to obtain the correct estimation of the radial wind velocity under the condition of a low SNR is to accumulate spectra recorded for single shots, which should result in smoothing of the noise peaks and amplification of the signal peak.

\subsection{Simulation of Retrieval of Vertical Wind Profiles from Accumulated Spectra}

Let the space-borne scanning coherent lidar provides us with the noised data approximated by the complex signal $Z\left(\theta_{i}, m T_{s}\right)$ to be further processed to retrieve the information about the wind. Here $\theta_{i}$ is the azimuthal scanning angle, and $i$ is the number of a sensing pulse. Since the bandwidth $B=50 \mathrm{MHz}, T_{s}=1 /(2 B)=10 \mathrm{~ns}$. To estimate the radial velocity at some fixed height, let us sample $M$ values of the recorded signal (vertical resolution).

Using a fast Fourier transform (FFT), we find the power spectra:

$W\left(\theta_{i}, \frac{k}{M T_{s}}\right)=\frac{T_{s}}{M}\left|\sum_{m=0}^{M-1} Z\left(\theta_{i}, m T_{s}\right) \exp [-2 \pi j(k m / M)]\right|^{2}$,

where $k=0,1, \ldots, M-1$. The frequency resolution here is determined by $\Delta f=1 /\left(M T_{s}\right)$. With allowance for the Doppler relation, the velocity resolution $\Delta V=(\lambda / 2) \Delta f$ for $M$ $=1024, \lambda=2 \mu \mathrm{m}$, and $T_{s}=10 \mathrm{~ns}$ is roughly $0.1 \mathrm{~m} / \mathrm{s}$.

Assume that $n$ single sensing pulses $(i=1,2, \ldots, n)$ fall within a cell of the horizontal GWS grid. If scanning is absent, then the procedure of data accumulation consists of simple summation of the spectra in the corresponding channels (numbers of the spectral channels $k=0,1, \ldots, M-1$ ). As a result, at the quite good averaging with the application
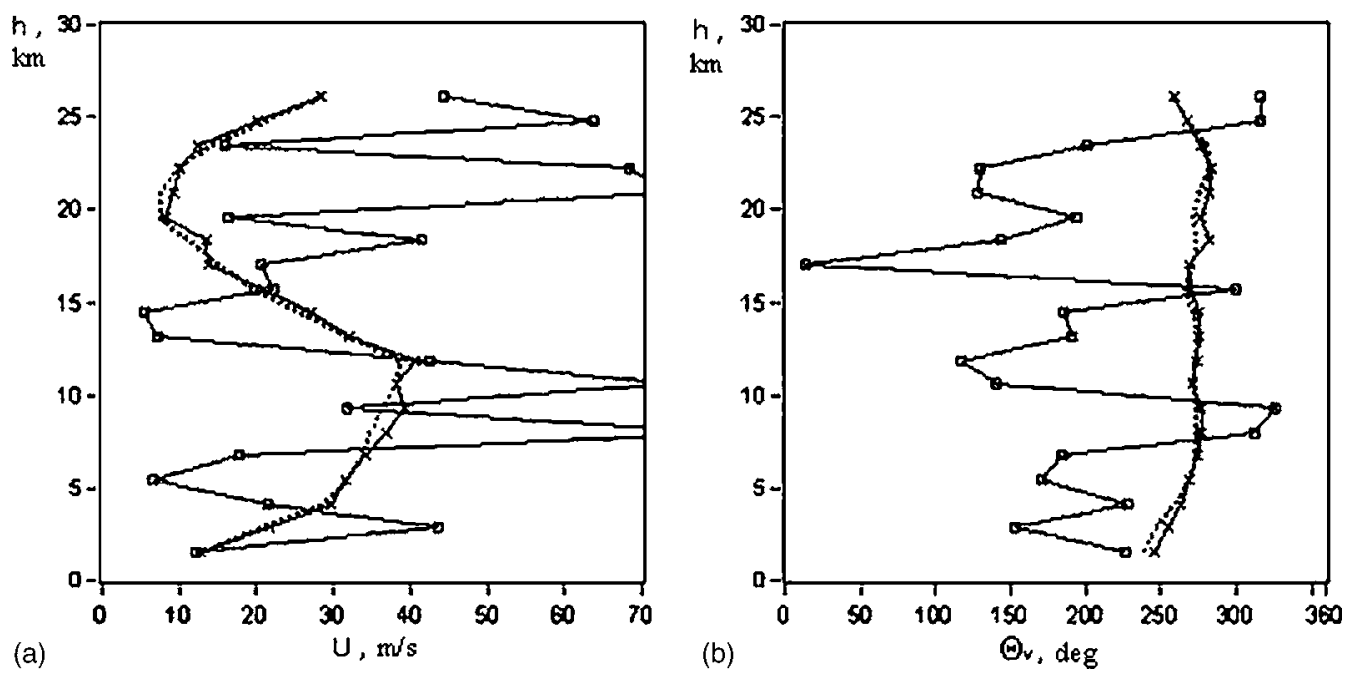

Fig. 8 Examples of the vertical profiles of (a) wind velocity and (b) direction as retrieved from the model lidar data with (squares) and without (crosses) regard for noise. GWS data are shown by dashed curves. 
of moving smoothing, the radial velocity can be estimated from the total spectrum with acceptable accuracy.

In our case of the scanning lidar, the radial wind velocity $V_{r}$ is a function of the angle $\theta_{i}$. On the assumption of the horizontally homogeneous wind $V_{r}\left(\theta_{i}\right)$ is determined by the equation

$V_{r}\left(\theta_{i}\right)=U \cos \alpha \cos \left(\theta_{i}-\theta_{V}\right)$

where $U$ and $\theta_{V}$ are the velocity and the angle of direction of a horizontal wind, $\alpha$ is the angle of elevation (angle between the axis of the sensing beam and the Earth's surface).

The wind parameters $U$ and $\theta_{V}$ can be found by omitting the stage of estimation of the radial velocity from the data of sensing by single pulses. ${ }^{59}$ With allowance for Eq. (43), we can calculate the 2-D array of the function

$F\left(U, \theta_{V}\right)=\sum_{i=1}^{n} \sum_{k^{\prime \prime}=k^{\prime}-\Delta k}^{k^{\prime}+\Delta k} W\left(\theta_{i}, \frac{k^{\prime \prime}}{M T_{s}}\right)$

where

$k^{\prime}=\left[\frac{U}{\Delta V} \cos \alpha \cos \left(\theta_{i}-\theta_{V}\right)\right]$,

$U=\Delta U l, \theta_{V}=\Delta \theta_{V} m, l=1,2, \ldots, m=1,2, \ldots, \Delta U$, and $\Delta \theta_{V}$ are the velocity and angular resolutions; the square brackets in Eq. (45) denote the integer part of a number. Then the wind velocity $U$ and direction $\theta_{V}$ are determined from the position of the peak of the function $F\left(U, \theta_{V}\right)$.

Thus, using Eq. (45), we estimate the wind velocity from the rather well averaged data: from $n$ sensing pulses and from the spectral window of the width $(2 \Delta k$ $+1) /\left(M T_{s}\right)$. Numerical experiments have shown that at the velocity resolution $\Delta V \approx 0.1 \mathrm{~m} / \mathrm{s}$ the value $\Delta k=4$ is optimal in Eq. (44).

According to the data of Fig. 7, a maximum of 53 pulses (on the average, $n \sim 26$ ) fall within the cell. From the results of numerical simulation, it follows that this number of pulses is enough to retrieve the wind profile up to the altitudes $\sim 7$ to $8 \mathrm{~km}$. The analysis of the GWS data for the wind at high altitudes has shown that for better averaging in Eq. (44) we can use the lidar data obtained with sensing pulses fallen within nine cells without fear of excessive averaging of the wind over the large area $\sim 300 \times 300 \mathrm{~km}$.

Figure 9 shows the examples of retrieval of the wind speed and direction by the method of spectrum accumulation in the cloudless and cloudy atmosphere along with the GWS data. We can see that in a clear sky, the wind profiles are retrieved with an acceptable accuracy up to the altitudes $\sim 18$ to $20 \mathrm{~km}$. In the presence of clouds, the retrieval error is quite large in the layer below $\sim 8 \mathrm{~km}$ because of the strong cloud extinction of the forward and backscattered radiation.

Based on the obtained data concerning the retrieval of the vertical wind profiles by the two methods-(1) the method in which first the estimates of the radial wind velocity are obtained and then the wind speed and direction are calculated from fitting of these estimates to the dependence described by Eq. (43) [let us call it the method of fitting of radial velocities (FRV)] and (2) the method of VAS described in this subsection-we calculated the absolute errors for the wind speed $\varepsilon_{U}=\left[\left\langle\left(U_{L}-U_{W}\right)^{2}\right\rangle\right]^{1 / 2}$ and direction $\varepsilon_{\theta}=\left[\left\langle\left(\theta_{L}-\theta_{W}\right)^{2}\right\rangle\right]^{1 / 2}$, where $U_{L}$ and $\theta_{L}$ are the lidar estimates, and $U_{W}$ and $\theta_{W}$ are the GWS data.

Figure 10 shows the part of the globe along the satellite track, for which the GWS data were used in numerical simulation. The values of $\varepsilon_{U}$ and $\varepsilon_{\theta}$ were calculated using 746 retrieved vertical profiles of the wind velocity and direction. We first calculated the errors $\varepsilon_{U}$ and $\varepsilon_{\theta}$ for individual wind profiles, and then obtained their values averaged over the sensed area shown in Fig. 10. In the cross section of the satellite track, the size of the sensed area is approximately $600 \mathrm{~km}$.

Figure 11 depicts the calculated altitude dependences of errors $\varepsilon_{U}$ and $\varepsilon_{\theta}$ in the cases of the FRV and VAS methods. The data were obtained on the assumption of the clear sky along the satellite track and with allowance for the cloud distribution (according to the GWS data for January 20, 1998) in the sensed area. From comparison of Figs. 11(a) and 11(b) with 11(c) and 11(d) it follows that in the cloudless atmosphere, the FRV method enables the profiles of the wind speed and direction to be retrieved with an acceptable accuracy up to the maximal altitudes of 2 to $3 \mathrm{~km}$, while the use of the VAS method makes it possible to retrieve the wind speed with the error $\lesssim 2 \mathrm{~m} / \mathrm{s}$ and the direction with the error $\lesssim 20 \mathrm{deg}$ up to the altitudes $\sim 18 \mathrm{~km}$. If clouds are present in the sensed area, then, as follows from Figs. 11(e) and $11(\mathrm{f})$, the lidar retrieval of the wind is possible only in the layer from 10 to $16 \mathrm{~km}$.

\section{Estimation of Turbulence Parameters from the Spatial Structure Function of Wind Velocity Measured by a Pulsed CDL}

In Sec. 2.2 it was shown that parameters of small-scale turbulence can be found from the spatial structure function of fluctuations of the cw Doppler lidar estimate of radial velocity measured under different azimuth angles [Eq. (22)]. In the case of pulsed Doppler systems, this can be done based on the spatial structure function of Doppler estimates of velocity at the points separated by distance $r$ along line of sight.

According to Ref. 51, the spatial structure function of fluctuations of the Doppler estimate of wind velocity $V_{D}(R)$ :

$D(r)=\left\langle\left[\widetilde{V}_{D}(R+r)-\tilde{V}_{D}(R)\right]^{2}\right\rangle$,

can be presented in the form

$D(r)=D_{a}(r)+D_{e}(r)$

where $\quad \tilde{V}_{D}(R)=V_{D}(R)-\left\langle V_{D}(R)\right\rangle=\tilde{V}_{a}(R)+\tilde{V}_{e}(R), \quad D_{a}(r)$ $=\left\langle\left[\widetilde{V}_{a}(R+r)-\tilde{V}_{a}(R)\right]^{2}\right\rangle$ is the spatial structure function of the radial wind velocity averaged over the sensed volume; $D_{e}(r)=\left\langle\left[\tilde{V}_{e}(R+r)-\tilde{V}_{e}(R)\right]^{2}\right\rangle$ is the structure function of the error in the estimated Doppler velocity due to fluctuations of the scattered wave and noise, $\tilde{V}_{a}$ and $\tilde{V}_{e}$ are independent. $^{30,32}$ 

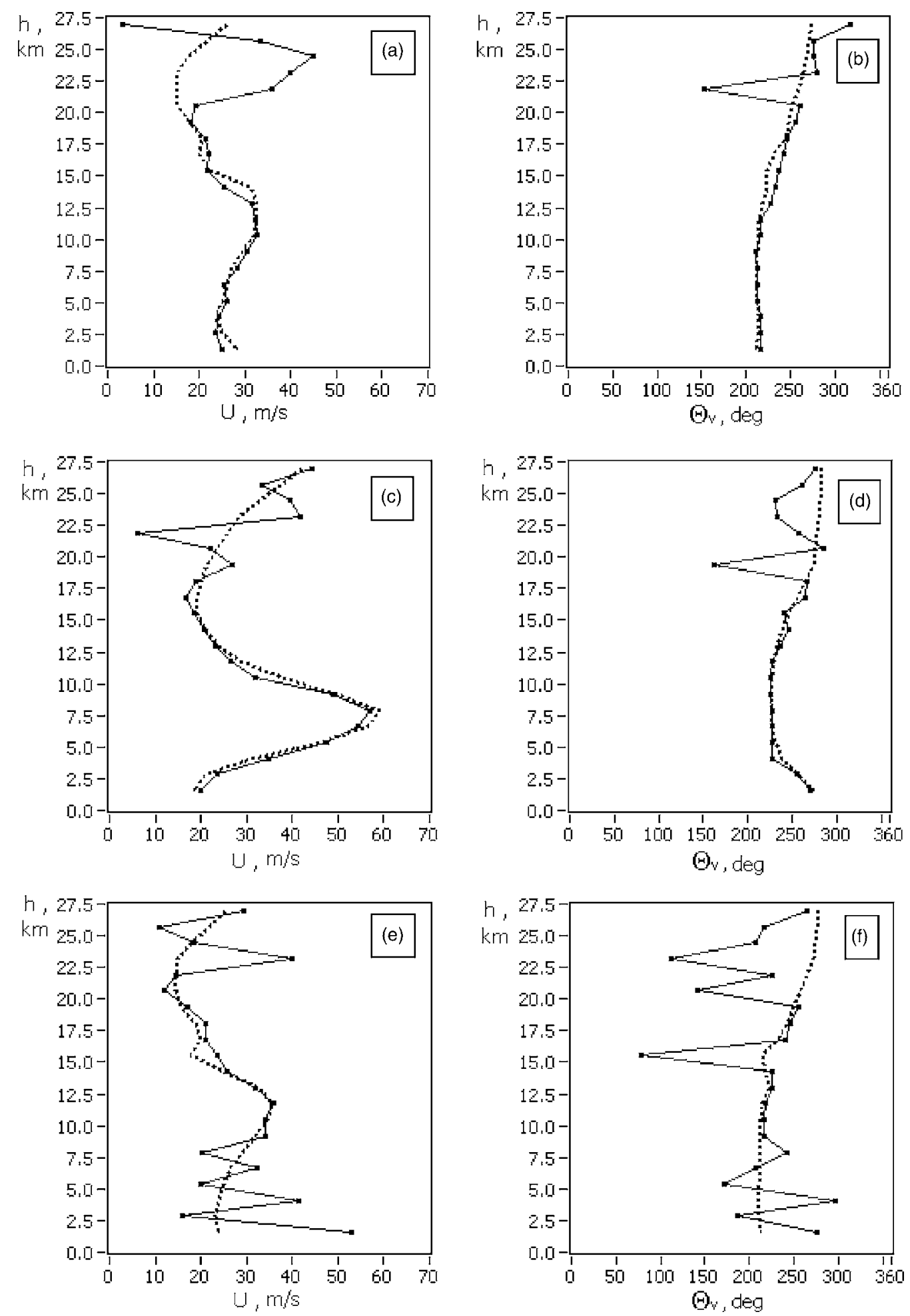

Fig. 9 Examples of retrieval of the (a), (c), and (e) wind velocity and (b), (d), and (f) direction by variational accumulation of spectra (VAS) in the (a) to (d) a cloudless atmosphere and (e) and (f) in the presence of clouds. Lidar data are shown by solid curves and GWS data by dashed curves. 


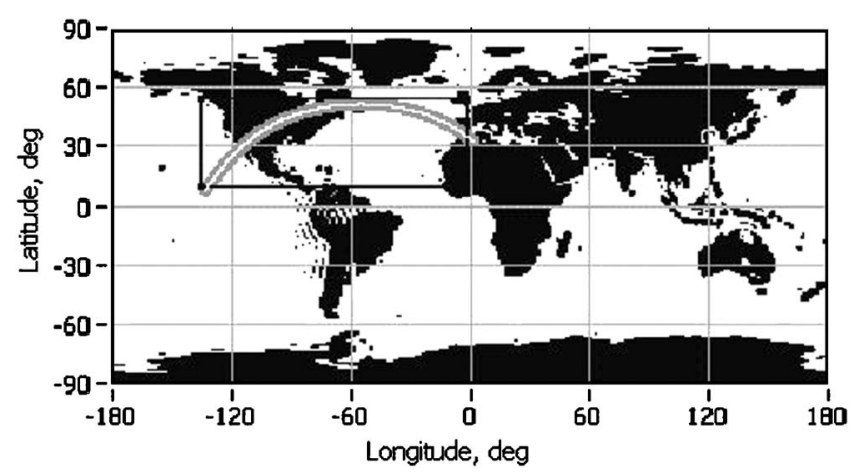

Fig. 10 Part of the globe along the satellite track (in frame) for which the GWS data were used in analysis of the errors of wind retrieval.

For the radial wind velocity averaged over the sensed volume, we can obtain ${ }^{51}$

$V_{a}(R)=\frac{1}{U_{p}} \int_{-\infty}^{\infty} \mathrm{d} t P_{T}(t) \frac{1}{\tau} \int_{-\tau / 2}^{\tau / 2} \mathrm{~d} t^{\prime} V_{r}\left[R+\frac{c}{2}\left(t+t^{\prime}\right)\right]$,

where $\tau=m T_{s}$. Hence for the structure function $D_{a}(r)$, we have

$$
\begin{aligned}
D_{a}(r)= & 2 \int_{-\infty}^{\infty} S_{r}(\kappa)[1-\exp (2 \pi j \kappa r)]\left(\frac{2}{\pi \kappa c \tau}\right)^{2} \sin ^{2}\left(\frac{\pi \kappa c \tau}{2}\right) \\
& \times \frac{1}{U_{p}^{2}} \int_{-\infty}^{\infty} \mathrm{d} t_{1} \int_{-\infty}^{\infty} \mathrm{d} t_{2} P\left(t_{1}\right) P\left(t_{2}\right) \exp \left[\pi j \kappa c\left(t_{1}-t_{2}\right)\right] .
\end{aligned}
$$

Represent the structure function of the error of the estimated Doppler velocity in the form

$D_{e}(r)=2\left[\sigma_{e}^{2}-B_{e}(r)\right]$,

where $B_{e}(r)=\left\langle\left[\tilde{V}_{e}(R+r) \tilde{V}_{e}(R)\right]\right\rangle$ and $\sigma_{e}^{2}=B_{e}(0)$ are the correlation function and the variance of the error of the estimated Doppler velocity, respectively.

If, following Refs. 32 and 51, we estimate the spatial structure function of the wind velocity from neighboring pulse shots:

$D_{p}(r)=\left\langle\left[\tilde{V}_{D}^{(1)}(R+r)-\tilde{V}_{D}^{(2)}(R)\right]\right\rangle$,

where the superscripts (1) and (2) correspond to the velocity estimates from returns of two neighboring shots, then, taking into account that $V_{a}(r), V_{e}^{(1)}(r)$, and $V_{e}^{(2)}(r)$ are independent, from Eq. (51) we have

$D_{p}(r)=2 \sigma_{e}^{2}+D_{a}(r)$,

where $2 \sigma_{e}^{2}=D_{p}(0)$.

Excluding the variance of the error of the estimate from Eq. (52), we obtain the estimate of the structure function of the radial wind velocity averaged over the sensed volume:

$\hat{D}_{a}(r)=D_{p}(r)-2 \sigma_{e}^{2}$.

\subsection{Wind Turbulence Parameter Estimation}

\subsubsection{2- $\mu \mathrm{m} C D L$}

The spatial statistics of the radial velocity estimates from the simulated and measured 2- $\mu \mathrm{m}$ CLD data is analyzed in Refs. 17 and 36 in detail for the Karman model of turbulence and arbitrarily spaced observation points. In this part, we are concerned with the results of estimation of the turbulent kinetic energy dissipation rate from the simulated Doppler velocity spatial structure function with a small separation of observation points, when the Kolmogorov spectrum is valid.

For $2-\mu \mathrm{m}$ pulsed CDLs, sensed volumes are not too large, as a rule, and their effective longitudinal dimension $\Delta z$ does not exceed the integral correlation scale of the wind velocity. In this case, to estimate the wind turbulence parameters and, in particular, the dissipation rate of the turbulent energy from lidar measurements of the wind velocity, it is possible to use the relations for the statistical characteristics of velocity fluctuations in the atmosphere, which are valid in the inertial range of turbulence. Actually, at small separations $r \ll L_{V}$ in Eq. (49), Eq. (15) can be used for $S_{r}(\kappa)$, so that the spatial structure function $D_{a}(r) \sim \varepsilon^{2 / 3}$. Then, based on Eq. (52), the dissipation rate $\hat{\varepsilon}$ can be estimated by the least-squares method.

The corresponding system of equations has the form

$\left\{\begin{array}{l}\frac{\partial \rho_{0}(\alpha, \beta)}{\partial \alpha}=0, \\ \frac{\partial \rho_{0}(\alpha, \beta)}{\partial \beta}=0,\end{array}\right.$

where

$\rho_{0}(\alpha, \beta)=\sum_{m=0}^{M^{\prime}}\left[\hat{D}_{P}(m \Delta r)-\alpha^{2}-\beta^{2} F_{a}(m \Delta r)\right]$,

$\Delta r=c T_{S} / 2$ is the distance between adjacent velocity estimates $M^{\prime} \Delta r \ll L_{V} ; \hat{D}_{P}(m \Delta r)$ is the estimate of the function of Eq. (52), $\quad \alpha=\sqrt{2} \hat{\sigma}_{e}, \quad \beta=(\hat{\varepsilon})^{1 / 3}, \quad$ and $\quad F_{a}(m \Delta r)$ $=D_{a}(m \Delta r) / \varepsilon^{2 / 3}$ is the function described by Eqs. (15), (24), and (49). In this case, we should select the real (nonzero at $\alpha^{2}>0$ and $\beta^{2}>0$ ) solutions of the system of Eqs. (54).

The modeling results at small values of SNR demonstrate the impossibility to obtain $\hat{\varepsilon}$ estimate with an acceptable accuracy from the function $\hat{D}_{P}(m \Delta r)$ measured in real time without the use of special procedures of data processing. The problem of a weak Doppler lidar signal regime is discussed in Ref. 31, 32, 60, and 61. In such a regime, we follow the procedure outlined in Ref. 32.

A simulation of the $2-\mu \mathrm{m}$ pulsed Doppler lidar data was carried out for typical parameters $\sigma=120 \mathrm{~ns}, M=16$, and $T_{S}=20 \mathrm{~ns}\left(\tau=M T_{S}=320 \mathrm{~ns}, c \tau / 2=\Delta z=48 \mathrm{~m}\right)$ and different SNRs. The velocity $V_{r}$ was simulated for the Karman model of spectrum [Eq. (14)] with $\sigma_{r}=1 \mathrm{~m} / \mathrm{s}$ and $L_{V}$ $=150 \mathrm{~m}$. In such a case, according to Eq. (16), the dissipation rate of the turbulent energy is $\varepsilon=4.45 \times 10^{-3} \mathrm{~m}^{2} / \mathrm{s}^{3}$. The length $L_{R}$ of random realizations of the radial wind velocity with zero mean $\left(\left\langle V_{r}\right\rangle=0\right)$ was $L_{R}=2048 \times \Delta p$ $=614.4 \mathrm{~m}$ with $\Delta p=0.3 \mathrm{~m}$. For each shot, on the assump- 

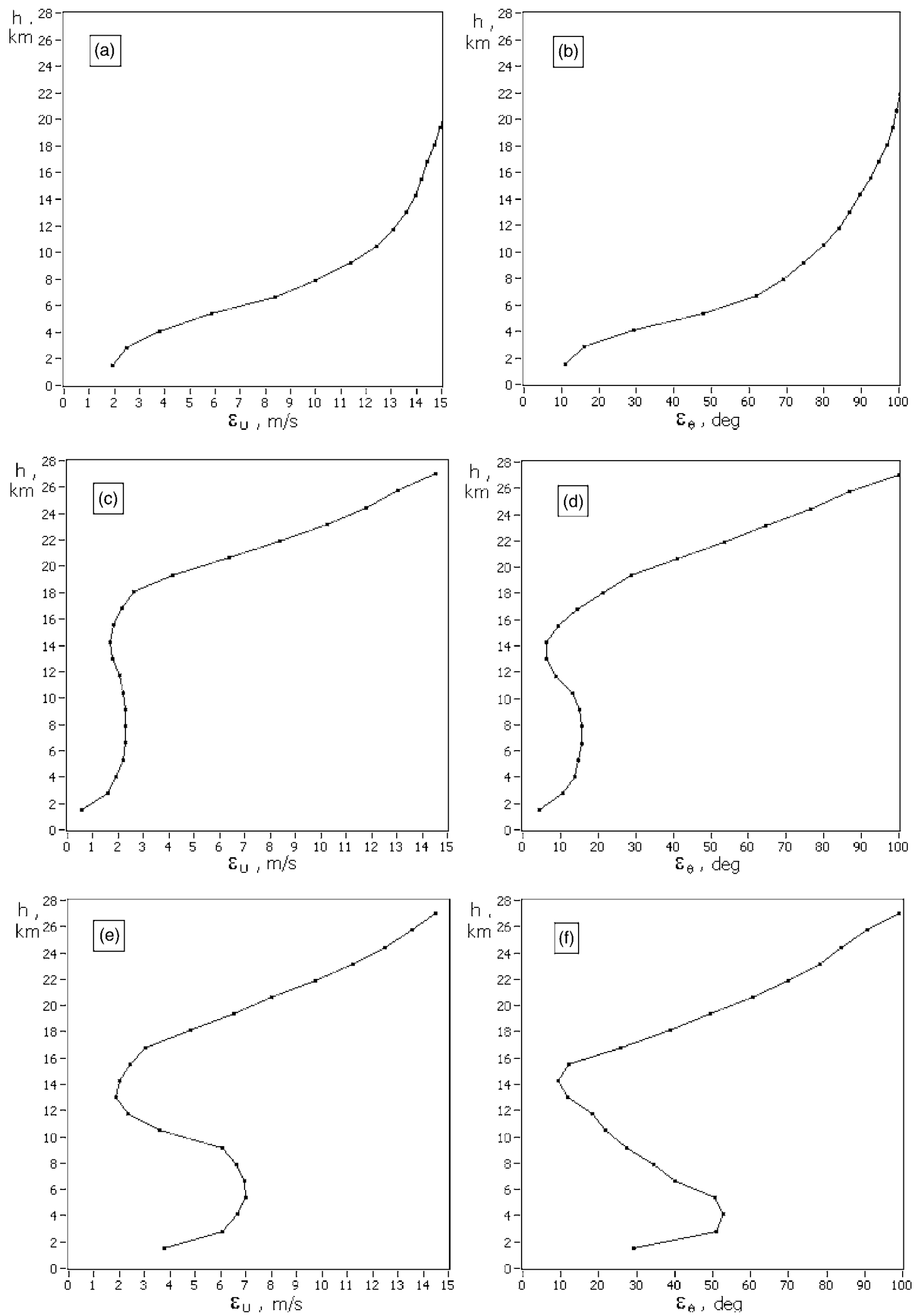

Fig. 11 Altitude dependence of the errors in estimation of the wind velocity $\varepsilon_{U}$ and direction $\varepsilon_{\theta}$ for the (a) and (b) FRV and (c) to (f) VAS methods, where (a) to (d) were calculated under clear-sky conditions and (e) and (f) were calculated with allowance for cloud distribution according to GWS data for January 20, 1998. 


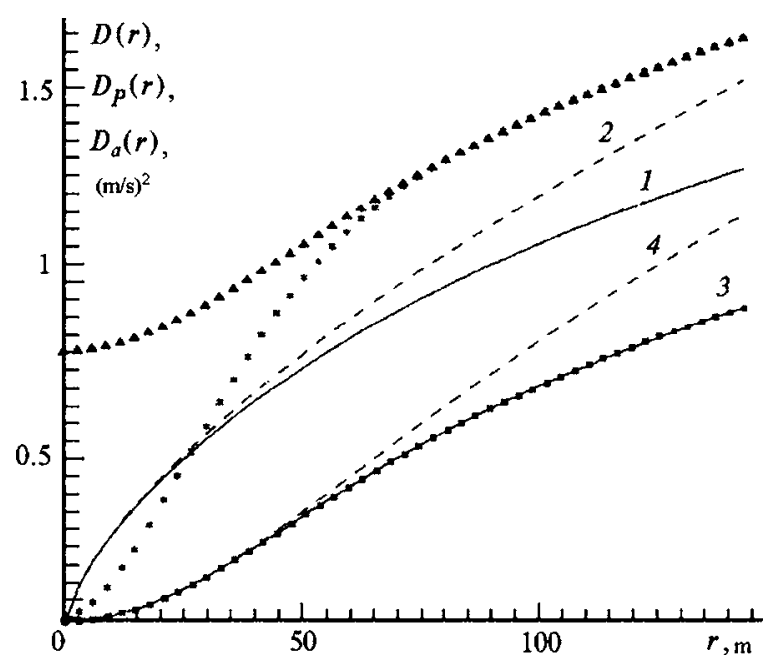

Fig. 12 Functions $D(r), D_{P}(r)$, and $D_{a}(r): 1$ and 2 correspond to the structure function $D(r)$ for the velocity measured by a point measurer in the case of the Karman (curve 1) and Kolmogorov (curve 2) spectra; 3 and 4 are calculations of the structure function $D_{a}(r)$ by Eq. (49) for the same models of the spectra, respectively; $\boldsymbol{\Lambda}, \mathbf{\square}$, *-results of simulation; $\boldsymbol{\Delta}-D_{P}(r) ; \boldsymbol{\square}-D_{a}(r) ; *-D(r)$.

tion that the pulse occupies the distance $\Delta L_{R}$ during observation time, 64 values of the complex signal $Z\left(m T_{S}\right)$ were simulated based on each random realization of the velocity and then used for estimation of 49 values of the velocity $V_{D}$ by the ACF method. Assuming that the shot repetition rate is $10 \mathrm{~Hz}$, we supposed that for the each subsequent shot the interval $\Delta L_{R}$ shifted by $0.9 \mathrm{~m}$ with respect to the distribution of the random realization of wind along the sensing path. For the outer scale of turbulence $L_{V}=150 \mathrm{~m}$, on the assumption of "frozen" turbulence, this corresponded to the correlation time of the radial velocity roughly equal to $15 \mathrm{~s}$. One realization of the wind $V_{r}$ of the length $L_{R}$ was used for 350 shots. For the results presented in the following, 1000 realizations of $V_{r}$ were simulated. The total number of the estimates of $V_{D}$ was $1000 \times 350 \times 49=1.715 \times 10^{7}$. In the case of low SNR when the large number of "bad" velocity estimates $^{32}$ are present, to obtain statistically valid estimates of the turbulent wind field parameters, an even greater number of sampled data were required.

Figure 12 shows the results of calculation of the functions $D(r), D_{P}(r)$, and $D_{a}(r)$ through computer simulation at $\mathrm{SNR}=100$ (signs) and calculation of $D(r)$ and $D_{a}(r)$ by Eq. (49) (solid and dashed curves). Curves 1 and 2 correspond to the calculated structure function of velocity measured by a point measurer for the Karman spectrum (14) (solid curve) and the Kolmogorov spectrum (15) (dashed curve). Curves 3 and 4 correspond to $D_{a}(r)$ calculated by Eq. (49) with allowance for averaging over the sensed volume. From a comparison of curves 1 and 2 (or 3 and 4) we can see that for the data presented the length of the inertial range of turbulence $r_{\text {in }}$, where these curves must be close, it is restricted to about $50 \mathrm{~m}$, that is, it is comparable with the longitudinal size of the sensed volume $r_{\text {in }} \approx \Delta z$. The discrepancy between curves 1 and 3 illustrates the effect of spatial averaging of the radial wind velocity over the sensed volume. With an increase of $r$, the difference be-

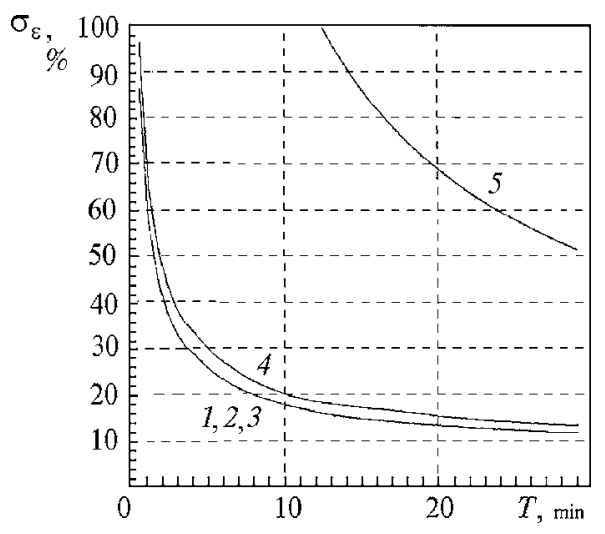

Fig. 13 Relative error in estimation of the dissipation rate of turbulent energy as a function of the averaging time for $S N R=1000$ (curve 1), 100 (curve 2), 10 (curve 3), 5 (curve 4), and 1 (curve 5).

tween $D_{P}(r)$ and $D(r)$ vanishes, which is caused by the decreasing of correlation between the errors $V_{e}(R+r)$ and $V_{e}(R)$ :

$D_{e}(R)=2\left[\sigma_{e}^{2}-B_{e}(r)\right] \underset{r \rightarrow \infty}{\rightarrow} 2 \sigma_{e}^{2}$.

From the obtained large array of $V_{D}$ values, we estimated the function $\hat{D}_{P}(m \Delta r)$ with the number of shots varied from 350 to 17,500. For the pulse repetition frequency of $10 \mathrm{~Hz}$, this corresponded to the averaging time $T$ from 35 to $1750 \mathrm{~s} \approx 30 \mathrm{~min}$. Then, using the values of $\hat{\varepsilon}$ found by the algorithm of Eqs. (54) and (55) and the value $\varepsilon$ $=4.45 \times 10^{-3} \mathrm{~m}^{2} / \mathrm{s}^{3}$ determined by the parameters $\sigma_{r}$ and $L_{V}$ [Eq. (16)] specified in simulation of random realizations of the radial velocity $V_{r}$ in accordance with the Karman spectrum [Eq. (14)], we calculated the dependence of the relative error in estimation of the dissipation rate $\sigma_{\varepsilon}=\langle(\hat{\varepsilon}$ $\left.-\varepsilon)^{2}\right\rangle^{1 / 2} / \varepsilon$ on the averaging time $T$. Figure 13 depicts the dependence of $\sigma_{\varepsilon}$ (in percent) on the averaging time $T$ for different SNRs. It can be seen that with the increase of $T$ the error $\sigma_{\varepsilon}$ decreases monotonically in the considered range of the averaging time and becomes lower than $20 \%$ already at 10 -min averaging.

\subsection{2 $\mathrm{CO}_{2} C D L$}

In the case of pulsed $\mathrm{CO}_{2} \mathrm{CDLs}$, the spatial resolution of wind measurements $\left(M T_{s} c / 2\right)$ is hundreds of meters, and the scattering volume can be comparable to or greater than the outer scale of turbulence. Due to the effect of the outer scale, the spatial structure function of wind velocity measured by the pulsed $\mathrm{CO}_{2} \mathrm{CDL} D_{a}(r)$, as a function of the separation distance $r$, differs from the dependence $D_{a}(r)$ characteristic for the inertial range.

Actually, it follows from Fig. 14 that the range of the separations $r$, at which the functions $D_{a}(r)$ calculated by Eq. (49) for the Kolmogorov [Eq. (15)] and Karman [Eq. (14)] spectra of wind velocity fluctuations coincide, is negligibly small. The calculations were performed for the time dependence of the pulse amplitude $P_{T}^{1 / 2}(t)$ shown in Fig. 6(a) and for the spatial resolution $c \tau / 2=\left(M T_{s} c\right) / 2=300$ and $768 \mathrm{~m}$. Thus, the determination of the dissipation rate 


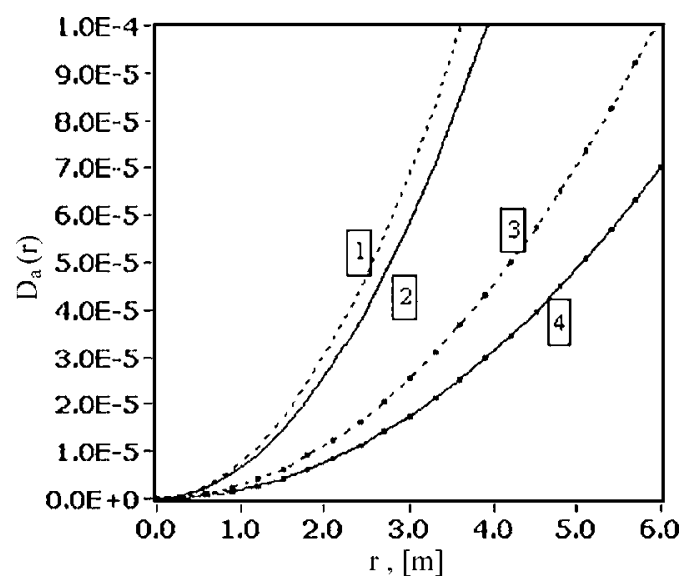

Fig. 14 Structure function $D_{a}(R)$ for the Kolmogorov and Karman spectra of turbulence calculated for measurements of the wind velocity with different spatial resolution. Curves 1 and 3 correspond to the Kolmogorov spectrum; curves 2 and 4 correspond to the Karman spectrum; $M=200$ (curves 1 and 2) and 512 (curves 3 and 4).

of turbulent energy, as in the case of the 2- $\mu \mathrm{m}$ lidar, from the estimate of the spatial structure function of the wind velocity at the separations $r \ll L_{V}$, turns out to be impossible for the pulsed $\mathrm{CO}_{2}$ CDLs. In this case, we must take into account the effect of the outer scale of turbulence.

The dissipation rate $\varepsilon$ can be found using Eq. (16), but the direct determination of the parameters $\sigma_{V}^{2}$ and $L_{V}$ from the spatial structure function of the wind is also impossible in this case, because, as follows from Eqs. (14) and (49), it depends on the product $\sigma_{V}^{2} L_{V}$, and the same value of the structure function can correspond to different combinations of the values of these parameters. To avoid the uncertainty in the parameters $\sigma_{V}^{2}$ and $L_{V}$, the following procedure is used.

Equation (49) is used to calculate the family of the structure functions $D_{a}\left(L_{V_{i}}, \sigma_{V}^{2} ; r\right)$ ( $r$ varies from 0 to $\left.r_{\max }\right)$ for the different values of the outer scale $L_{V_{i}}$. The value of the variance $\sigma_{V}^{2}$ in Eq. (49) can be arbitrary $\left(\sigma_{V_{c}}^{2} \equiv \sigma_{V}^{2}=\right.$ const). The choice of $r_{\max }$ is determined by the maximal separation at which, in the experiment, it is still possible to obtain the estimate of the structure function [Eq. (53)]. The value of $r_{\max }$ should be large enough for the structure function to saturate at $r_{\max }$, that is, to weakly depend on the separation. This enables elimination of the parameter $\sigma_{V}^{2}$ through normalization of $D_{a}\left(L_{V_{i}} ; \sigma_{V_{c}}^{2} ; r\right)$ to $D_{a}\left(L_{V_{i}} ; \sigma_{V_{c}}^{2} ; r_{\max }\right)$ :

$\overline{D_{a}\left(L_{V_{i}} ; r\right)}=\frac{D_{a}\left(L_{V_{i}} ; \sigma_{V_{c}}^{2} ; r\right)}{D_{a}\left(L_{V_{i}} ; \sigma_{V_{c}}^{2} ; r_{\max }\right)}$.

Figure 15 exemplifies the family of the normalized structure functions [Eq. (56)] calculated for different values of $L_{V}$. If the experimental estimate of the structure function $\hat{D}_{a}(r) \quad[$ Eq. $\quad(53)]$ is normalized to $\hat{D}_{a}\left(r_{\max }\right): \hat{\hat{D}}_{a}(r)$ $=\left[\hat{D}_{a}(r)\right] /\left[\hat{D}_{a}\left(r_{\text {max }}\right)\right]$, then we can use the parametric fitting method and estimate the integral scale of wind velocity fluctuations $\hat{L}_{V}$ through minimization of the functional:

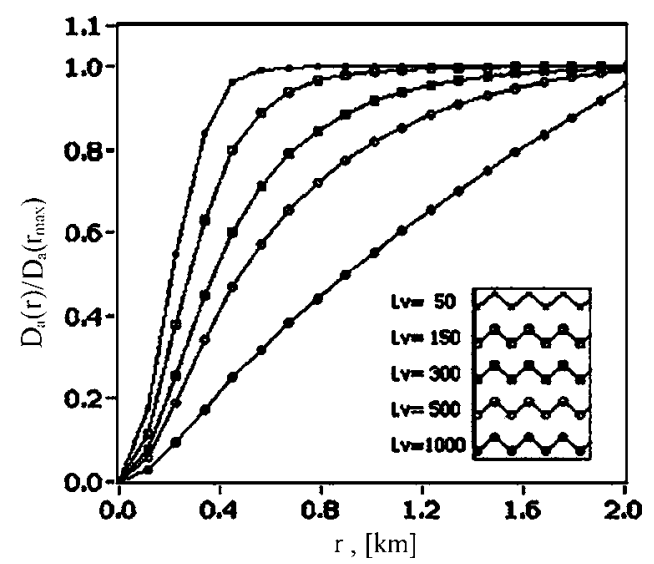

Fig. 15 Family of normalized structure functions calculated for different values of the integral scale $L_{V} ; M=512$.

$\sum_{i}\left[\overline{\hat{D}_{a}(r)}-\overline{D_{a}\left(L_{V_{i}}, r\right)}\right]^{2}$

Using the estimated scale $\hat{L}_{V}$, we can estimate the variance $\hat{\sigma}_{V}^{2}$ :

$\frac{\hat{\sigma}_{V}^{2}}{\sigma_{V_{c}}^{2}}=\frac{\hat{D}_{a}\left(r_{\max }\right)}{D_{a}\left(L_{V_{i}}, \sigma_{V_{c}}^{2}, r_{\max }\right)}$.

The dissipation rate of turbulent energy $\hat{\varepsilon}$ is estimated from the obtained estimates of the variance $\hat{\sigma}_{V}^{2}$ and the scale $\hat{L}_{V}$ by Eq. (16).

The signal of the 10.6- $\mu \mathrm{m}$ pulsed Doppler lidar was simulated numerically using the real records of the $\mathrm{CO}_{2}$ laser pulses of the WIND system [Fig. 6(b)] for $T_{S}$ $=10^{-8} \mathrm{~s}$ and different values of the SNR. Individual random realizations of the wind velocity were generated for the case of the Karman spectrum [Eq. (14)] at $\sigma_{V}=1 \mathrm{~m} / \mathrm{s}$ and $L_{V}=500 \mathrm{~m}$. In this case, according to Eq. (16), the dissipation rate of turbulent energy was $\varepsilon=1.33$ $\times 10^{-3} \mathrm{~m}^{2} / \mathrm{s}^{3}$. The sample included 4096 values of the wind velocity with the separation $\Delta p=1.5 \mathrm{~m}$ between readings. For each shot, 2048 values were simulated, that is, only a half of the random velocity realization was used. The pulse repetition frequency was taken as $10 \mathrm{~Hz}$, and it was assumed that the wind realization shifted by $1.5 \mathrm{~m}$ for the each subsequent shot. Thus, one realization of the wind $V_{r}$ was used for simulation of 1024 shots.

From the signal series $Z\left(m T_{S}\right), M=200$ and $M=512$ values were sampled, which corresponded to the spatial resolution of 300 and $768 \mathrm{~m}$, and the velocity $V_{D}$ was estimated by the ACF method. For one realization of the wind velocity, we obtained 1849 estimates of $V_{D}$ for $M=300$ and 1537 estimates for $M=512$. The total number of lidar estimates of the velocity obtained from one wind realization was $1024 \times 1849$ if $M=300$ and $1024 \times 1537$ if $M=512$. A total of 960 realizations of the wind velocity were used.

The structure function $\hat{D}_{a}(r)$ was estimated from the large array of $V_{D}$ data with variation of the number of shots from 600 to 18,000 . For the pulse repetition frequency of $10 \mathrm{~Hz}$, this corresponded to the averaging time $T$ from 1 to 

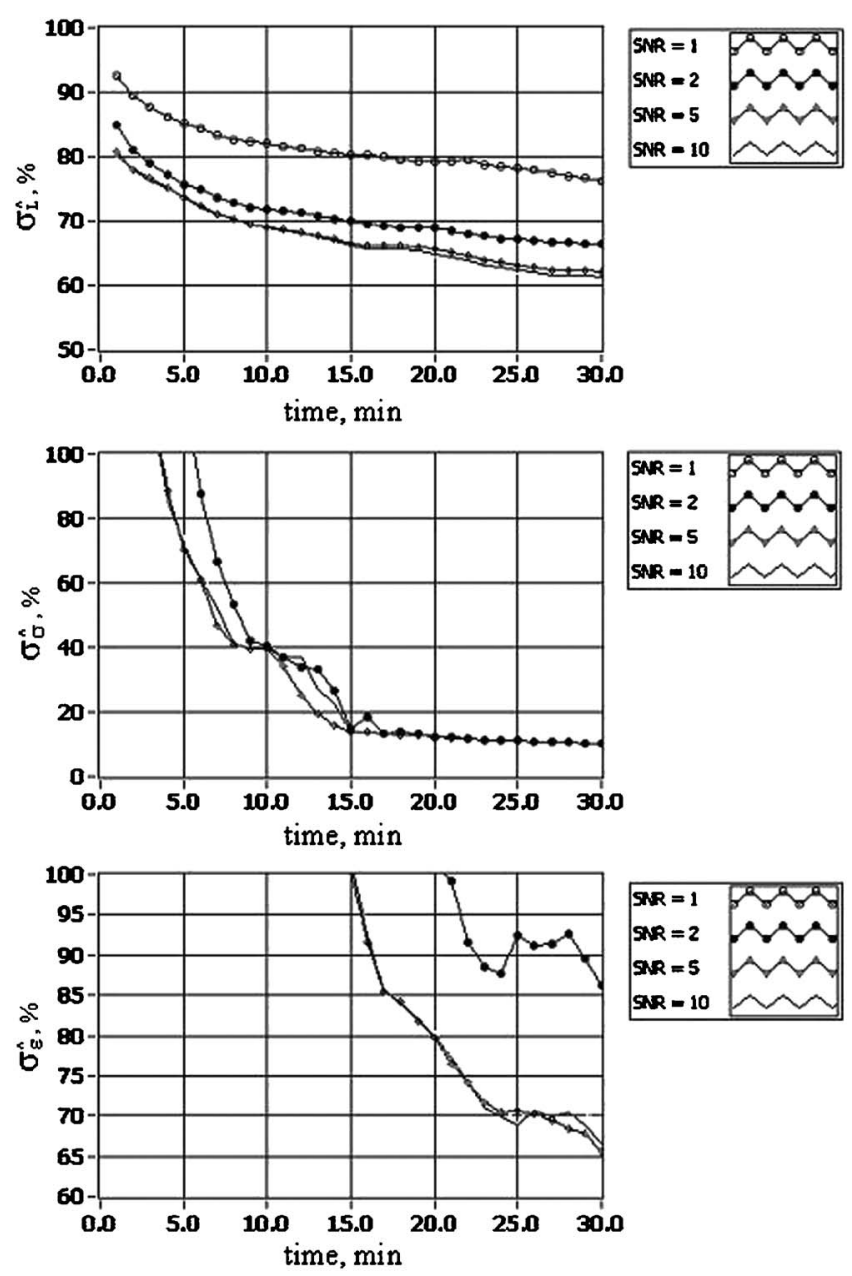

Fig. 16 Relative errors in measurement of the turbulence parameters; $M=200$ and spatial resolution is $300 \mathrm{~m}$.

$30 \mathrm{~min}$. Then, the estimates $\hat{L}_{V}, \hat{\sigma}_{V}^{2}$, and $\hat{\varepsilon}$ were determined by the algorithm of Eqs. (56)-(58) and (16). From comparison of the estimates $\hat{L}_{V}, \hat{\sigma}_{V}^{2}$, and $\hat{\varepsilon}$ with the values of these parameters specified in simulation of the turbulent wind field, the dependences of the relative errors in estimation of the turbulent parameters on the averaging time $T$ were calculated. The results are presented in Fig. 16. We can see from the figure that for the $S N R \geqslant 2$, the relative error of retrieval of the parameters $\sigma_{V}^{2}$ and $\varepsilon$ from the lidar data lies in the range $<50 \%$ already for the 5-min averaging, which is quite acceptable for geophysical measurements. The situation with the integral correlation scale of the wind velocity is worse. In this case, the relative error corresponding to the 5-min averaging is 70 to $80 \%$ and decreases very slowly with the increasing averaging time.

\section{Conclusions}

We described algorithms for simulating the operation of CDLs employing $\mathrm{cw}$ and pulsed laser sources along with the methods for estimation of the mean wind and the parameters of small-scale wind turbulence from the radial velocity measured with CDL. The lidar measurement of the wind velocity in the turbulent atmosphere was simulated numerically and the errors of estimation of the turbulent parameters and retrieval of the vertical profiles of the speed and direction of the mean wind from the model lidar data were calculated.

The combination of the algorithms presented in this paper, the methods of computer simulation, and the corresponding LabView programs essentially form the virtual instrument emulating the CDL operation and enabling efficient, statistically justified planning of experiments on measurement of the parameters of the turbulent wind field in the atmosphere.

\section{Acknowledgments}

The support from the Russian Foundation for Basic Research Grant No. 03-05-64194 is acknowledged.

\section{References}

1. S. W. Henderson, C. P. Hale, J. R. Magee, M. J. Kavaya, and A. V. Huffaker, "Eye-safe coherent laser radar system at $2.1 \mu \mathrm{m}$ using Tm. Ho:YAG lasers," Opt. Lett. 16, 773-775 (1991).

2. S. W. Henderson, P. J. M. Suni, C. P. Hale, S. M. Hannon, J. R. Magee, D. L. Bruns, and E. H. Yuen, "Coherent laser radar at $2.1 \mu \mathrm{m}$ using solid-state lasers," IEEE Trans. Geosci. Remote Sens. GE-31, 4-15 (1993)

3. S. F. Clifford, J. C. Kaimal, R. J. Lataitis, and R. G. Strauch, "Ground-based remote profiling in atmospheric studies: An overview," Proc. IEEE 82, 313-355 (1994).

4. M. R. Huffaker and R. M. Hardesty, "Remote sensing of atmospheric wind velocities using solid-state and $\mathrm{CO}_{2}$ coherent laser systems," Proc. IEEE 84, 181-204 (1996).

5. J. M. Vaughan, O. Steinvall, C. Werner, and P. H. Flamant, "Coherent laser radar in Europe," Proc. IEEE 84, 205-226 (1996).

6. J. M. Wilczak, E. E. Gossard, W. D. Neff, and W. L. Eberhard, "Ground-based remote sensing of the atmospheric boundary layer: 25 years of progress," Boundary-Layer Meteorol. 78, 321-349 (1996).

7. J. Rothermel, C. Kessinger, and D. L. Davis, "Dual-Doppler lidar measurement of winds in the JAWS experiment," J. Atmos. Ocean. Technol. 2, 138-147 (1985).

8. C. Werner, P. H. Flamant, O. Reitebuch, F. Kopp, J. Streicher, S. Rahm, E. Nagel, M. Klier, H. Herrmann, C. Loth, P. Delville, P. Drobinski, B. Romand, C. Boitel, D. Oh, M. Lopez, M. Meissonnier, D. Bruneau, and A. Dabas, "Wind infrared Doppler lidar instrument," Opt. Eng. 40, 115-125 (2001).

9. O. Reitebuch, C. Werner, I. Leike, P. Delville, P. Flamant, A. Cress, and D. Engelbart, "Experimental validation of wind profiling performed by the airborne $10 \mu \mathrm{m}$-heterodyne Doppler lidar WIND," $J$. Atmos. Ocean. Technol. 18, 1331-1344 (2001).

10. H. R. Schulte, "Atmospheric Dynamic Mission: Project status, concepts review and technical baseline," in Proc. 10th Coherent Laser Radar Conf., pp. 94-97, Mt. Hood, OR (1999).

11. G. D. Emmitt, M. Kavaya, and T. Miller, "Sparcle: A mission overview," in Proc. 10th Coherent Laser Radar Conf., pp. 88-93, Mt. Hood, OR (1999).

12. G. M. Ancellet, R. T. Menzies, and W. B. Grant, "Atmospheric velocity spectral width measurements using the statistical distribution of pulsed $\mathrm{CO}_{2}$ lidar return signal intensities," J. Atmos. Ocean. Technol. 6, 50-58 (1989).

13. W. L. Eberhard, R. E. Cupp, and K. R. Healy, "Doppler lidar measurements of profiles of turbulence and momentum flux," J. Atmos. Ocean. Technol. 6, 809-819 (1989).

14. T. Gal-Chen, M. Xu, and W. L. Eberhard, "Estimations of atmospheric boundary layer fluxes and other turbulence parameters from Doppler lidar data," J. Geophys. Res. 97, 18409-18423 (1992).

15. V. A. Banakh, F. Kopp, C. Werner, and I. N. Smalikho, "Measurement of turbulent energy dissipation rate with a cw Doppler lidar in the atmospheric boundary layer," J. Atmos. Ocean. Technol. 16(8), 1044-1061 (1999).

16. R. Frehlich, S. Hannon, and S. Henderson, "Coherent Doppler lidar measurements of winds in the weak signal regime," Appl. Opt. 36, 3491-3499 (1997).

17. R. Frehlich, S. Hannon, and S. Henderson, "Coherent Doppler lidar measurements of wind field statistics," Boundary-Layer Meteorol. 86, 233-256 (1998).

18. P. Drobinski, A. Dabas, and P. H. Flamant, "Remote measurement of turbulent wind spectra by heterodyne Doppler lidar technique," $J$. Appl. Meteorol. 39, 2434-2451 (2000).

19. K. E. Bozier and C. G. Collier, "Observations of boundary layer turbulence spectra and kinetic energy dissipation using Doppler lidar," in Proc. 11th Coherent Laser Radar Conf., pp. 206-209, Mal- 
vern, UK (2001)

20. S. Bastin, P. Drobinski, A. Dabas, O. Reitebuch, P. Delwille, Ch Werner, A. Delaval, Ch. Boitel, H. Herrmann, E. Nagel, B. Romand, J. Streicher, B. Benech, O. Bock, J. L. Caccia, P. Durand, and V. Guenard, "Analysis of IOP2b land-sea breeze case during the ESCOMPTE experiment," in Lidar remote sensing. Papers 21st Int. Laser Radar Conf., L. R. Bissonnette, G. Roy, and G. Vallee, Eds., pp. 849-852, Quebec, Canada (2002).

21. O. Reitebuch, H. Volkert, Ch. Werner, A. Dabas, P. Delville, P. Drobinski, P. H. Flamant, and E. Richard, "Determination of air flow across the Alpine ridge by a combination of airborne Doppler lidar, routine radio-sounding and numerical simulation," $Q$. J. R. Meteorol. Soc. 129, 715-728 (2003).

22. S. M. Hannon, P. Gatt, S. W. Henderson, and R. M. Huffaker, "Recent progress and measurement results with commercial $2 \mu \mathrm{m}$ Doppler lidar," in Proc. 12th Coherent Laser Radar Conf., pp. 86-89 (2003).

23. O. Reitebuch, C. Werner, A. Dabas, P. Delville, P. Drobinski, and L. Gantner, "Airborne Doppler lidar observations and numerical simulations of 'alpine pumping' mesoscale flow," in Proc. 12th Coherent Laser Radar Conf., pp. 102-105 (2003).

24. V. Banakh, C. Werner, J. Streicher, O. Reitebuch, E. Nagel, T. Schneiderhan, T. Konig, S. Lehner, A. Dabas, and P. Delville, "Wind turbulence parameters measurements over sea by Doppler lidar and radar," in Proc. 12th Coherent Laser Radar Conf., pp. 191-196 (2003).

25. R. M. Hardesty, W. A. Brewer, S. P. Sandberg, C. J. Senff, B. J. McCarty, A. M. Weickmann, G. Ehret, A. Fix, G. Poberaj, M. Wirth, and C. Kiemle, "Airborne measurements of boundary layer turbulent structures using co-deployed Doppler and DIAL lidars," in Proc. 12th Coherent Laser Radar Conf., pp. 182-185 (2003).

26. F. Davies, C. G. Collier, G. N. Pearson, and K. E. Bozier, "Doppler lidar measurements of turbulent structure function over an urban area," J. Atmos. Ocean. Technol. 21, 753-761 (2004).

27. R. G. Frehlich and M. J. Kavaya, "Coherent laser radar performance for general atmospheric refractive turbulence," Appl. Opt. 30(36), 5325-5352 (1991)

28. R. G. Frehlich, "Doppler lidar signal covariance including wind shear and wind turbulence," Appl. Opt. 33(27), 6472-6481 (1994).

29. R. G. Frehlich and M. J. Yadlowsky, "Performance of meanfrequency estimators for Doppler radar and lidar," J. Atmos. Ocean. Technol. 11, 1217-1230 (1994).

30. R. G. Frehlich, S. M. Hannon, and S. W. Henderson, "Performance of a $2 \mu \mathrm{m}$ coherent Doppler lidar for wind measurement," J. Atmos. Ocean. Technol. 11(6), 1517-1528 (1994).

31. R. G. Frehlich, "Simulation of coherent Doppler lidar performance in the weak signal regime," J. Atmos. Ocean. Technol. 13, 646-658 (1996).

32. R. G. Frehlich, "Effects of wind turbulence on coherent Doppler lidar performance," J. Atmos. Ocean. Technol. 14, 54-75 (1997).

33. R. G. Frehlich and L. Cornman, "Coherent Doppler lidar signal spectrum with wind turbulence," Appl. Opt. 38(36), 7456-7466 (1999).

34. R. G. Frehlich, "Simulation of coherent Doppler lidar performance for space-based platforms," J. Appl. Meteorol. 39, 245-262 (2000).

35. R. G. Frehlich, "Errors for space-based Doppler lidar wind measurements: Definition, performance, and verification," J. Atmos. Ocean. Technol. 18, 1749-1772 (2001).

36. R. G. Frehlich and L. Cornman, "Estimating spatial velocity statistics with coherent Doppler lidar," J. Atmos. Ocean. Technol. 19, 355-366 (2002).

37. R. G. Frehlich, "Performance of coherent Doppler lidar velocity estimates with pulse accumulation," in Proc. 12th Coherent Laser Radar Conf., pp. 139-142 (2003).

38. R. G. Frehlich," "Velocity error for coherent Doppler lidar with pulse accumulation," J. Atmos. Ocean. Technol. 21, 905-920 (2004).

39. C. M. Sonnenschein and F. A. Horrigan, "Signal-to-noise relationships for coaxial systems that heterodyne backscatter from the atmo- sphere," Appl. Opt. 10, 1600-1604 (1971).

40. T. R. Lawrence, D. J. Wilson, C. E. Craver, I. P. Jones, R. M. Huffakcr, and J. A. Thomson, "A laser velocimeter for remote wind sensing," Rev. Sci. Instrum. 43, 512-518 (1972).

41. I. N. Smalikho, "On measurement of the dissipation rate of the turbulent energy with a cw Doppler lidar," Atmos. Oceanic Opt. 8(10), $788-793$ (1995).

42. V. A. Banakh and I. N. Smalikho, "Determination of the turbulent energy dissipation rate from lidar sensing data," Atmos. Oceanic Opt. 10(4-5), 295-302 (1997).

43. V. A. Banakh, C. Werner, and I. N. Smalikho, "Simulation of sounding of the atmospheric wind fields by Doppler lidar systems," in Application of the Conversion Research Results for International Cooperation. Sibconvers'99, Proc. IEEE 99EX246, 380-382 (1999).

44. N. K. Vinnichenko, N. Z. Pinus, S. M. Shmeter, and G. N. Shur Turbulence in the Free Atmosphere, Consultants Bureau, New York (1976).

45. A. S. Monin and A. M. Yaglom, Statistical Fluid Mechanics: Mechanics of Turbulence, Part 2, The MIT Press, New York (1981).

46. J. L. Lumley and H. A. Panofsky, The Structure of Atmospheric Turbulence, Wiley Interscience, New York (1964).

47. V. V. Bykov, Digital Simulation in Statistical Radiotechnique, Sovetskoe radio, Moscow (1971) (in Russian).

48. V. M. Gordienco, A. A. Kormakov, L. A. Kosovsky, N. N. Kurochkin, G. A. Pogosov, A. V. Priezzhev, and Y. Y. Putivskii, "Coherent $\mathrm{CO}_{2}$ lidars for measuring wind velocity and atmospheric turbulence," Opt. Eng. 33, 3206-3213 (1994).

49. V. A. Banakh, C. Werner, F. Kopp, and I. N. Smalikho, "Measurement of the turbulent energy dissipation rate with a scanning Doppler lidar," Atmos. Oceanic Opt. 9(10), 849-853 (1996).

50. V. A. Banakh, I. N. Smalikho, F. Kopp, and C. Werner, "Representativity of the wind measurements by a cw Doppler lidar in the atmospheric boundary layer," Appl. Opt. 34(12), 2055-2067 (1995).

51. V. A. Banakh and I. N. Smalikho, "Estimation of the turbulence energy dissipation rate from the pulsed Doppler lidar data," Atmos. Oceanic Opt. 10(12), 957-965 (1997).

52. V. A. Banakh and V. L. Mironov, Lidar in a Turbulent Atmosphere, Artech House, Boston, London (1987).

53. A. Van Der Ziel, Noise in Measurements, Wiley, New York (1975).

54. I. Leike, J. Streicher, C. Werner, V. A. Banakh, I. N. Smalikho, W. Wergen, and A. Cress, "Virtual Doppler lidar instrument," J. Atmos. Ocean. Technol. 18, 1447-1456 (2001).

55. K. V. J. Potty, U. C. Mohanty, and S. Raman, "Effect of three different boundary-layer parameterisations in a regional atmospheric model on the simulation of summer monsoon circulation," BoundaryLayer Meteorol. 84, 363-381 (1997).

56. M. Beniston, From Turbulence to Climate, Springer-Verlag, Berlin, Heidelberg, New York (1998).

57. L. T. Matveev, Course of General Meteorology, Atmospheric Physics, Student's Book, Gidrometeoizdat, Leningrad (1984) (in Russian)

58. H. A. Panofsky and J. A. Dutton, Atmospheric Turbulence. Models and Methods for Engineering Application, Wiley-Interscience, New York, Singapore (1984).

59. V. A. Banakh, C. Werner, W. Wergen, A. Cress, N. P. Krivolutskii, I. Leike, I. N. Smalikho, and J. Streicher, "Modeling of wind reconstruction from measurements with a space-borne coherent Doppler lidar," Atmos. Oceanic Opt. 14(10), 848-855 (2001).

60. B. J. Rye and R. M. Hardesty "Discrete spectral peak estimation in incoherent backscatter heterodyne lidar. 1. Spectral accumulation and the Cramer-Rao lower bound," IEEE Trans. Geosci. Remote Sens. GE-31, 16-27 (1993).

61. B. J. Rye and R. M. Hardesty, "Discrete spectral peak estimation in incoherent backscatter heterodyne lidar. II. Correlogram accumulation," IEEE Trans. Geosci. Remote Sens. GE-31, 28-35 (1993).

Biographies and photographs of authors not available. 